\title{
Efficacy and Safety of Afatinib in the Treatment of Advanced Non-Small-Cell Lung Cancer with EGFR Mutations: A Meta-Analysis of Real-World Evidence
}

\author{
Lemeng Zhang $(\mathbb{D}$, Yongzhong Luo $\mathbb{D}$, Jianhua Chen, Tianli Cheng, Hua Yang, \\ Changqie Pan, Haitao Li, and Zhou Jiang
}

Thoracic Medicine Department 1, Hunan Cancer Hospital, Changsha 410013, Hunan Province, China

Correspondence should be addressed to Yongzhong Luo; luoyongzhong@hnca.org.cn

Received 21 October 2021; Accepted 29 November 2021; Published 18 December 2021

Academic Editor: Ozkan Kanat

Copyright ( 92021 Lemeng Zhang et al. This is an open access article distributed under the Creative Commons Attribution License, which permits unrestricted use, distribution, and reproduction in any medium, provided the original work is properly cited.

\begin{abstract}
Introduction. The purpose of this study was to explore the efficacy and safety of afatinib in advanced non-small-cell lung cancer (NSCLC) patients with epidermal growth factor receptor (EGFR) mutations based on real-world evidence. Materials and Methods. Eligible real-world studies were identified from PubMed, Cochrane Library, and Embase. Cochrane guidelines were used to assess the quality of included studies. Cochran's $Q$ test and $\mathrm{I}^{2}$ statistics were used for the heterogeneity analysis. Results. Twenty-five studies were included in this meta-analysis; nine studies were included in the qualitative descriptive analysis. The summarized disease control rate (DCR) was $87.6 \%$ (81.5\%, 92.7\%), and the overall response rate (ORR) was $58.9 \%(48.8 \%$, 68.7\%). The pooled median progression-free survival (PFS) was $12.4(10.3,14.5)$ months, mean time to failure (TTF) was $15.4(13.6,17.2)$ months, and median overall survival (OS) was $31.6(26.7,36.5)$ months. The total incidences of adverse events (AEs) for skin rashes, diarrhea, paronychia, and mucositis were 71.4\% (64.4\%, 77.9\%), 70.4\% (60.1\%, 79.8\%), 52.1\% (41.9, 62.3\%), and 36.5\% (29.5\%, 43.8\%), respectively. The incidences of severe adverse events (SAEs, Grade $\geq 3$ ) for diarrhea, skin rashes, paronychia, and mucositis were 9.7\% (6.8\%, 13.1\%), 5.8\% (4.5\%, 7.2\%), 3.8\% (2.0\%, 6.2\%), and 2.1\% (1.0\%, 3.6\%), respectively. Differences in PFS and OS between the afatinib non-full-dose $(<40 \mathrm{mg})$ and full-dose $(>40 \mathrm{mg})$ groups were not significant $(P>0.05)$. However, the ORR in the fulldose group was $78.5 \%(66.7 \%, 88.4 \%)$, which was significantly higher than that in the non-full-dose group (67.8\% [56.8\%, 77.9\%]). Conclusion. The efficacy and safety of afatinib has been confirmed by real-world evidence in advanced NSCLC with EGFR mutation, consistent with randomized controlled trial results. In real-world setting, tolerability-guided dose adjustment might not affect the afatinib efficacy.
\end{abstract}

\section{Introduction}

Lung cancer is the leading cause of cancer-related deaths and a serious threat to human health [1]. Non-small-cell lung cancer (NSCLC) accounts for more than $80 \%$ of lung cancer [2]. Epidermal growth factor receptor (EGFR) mutations have been identified in approximately $50 \%$ of Asian and $10-15 \%$ of Caucasian lung adenocarcinoma patients $[3,4]$. Currently, tyrosine kinase inhibitors (TKIs), including erlotinib, gefitinib, dacomitinib, afatinib, and osimertinib, are the standard first-line treatment for advanced NSCLC patients with EGFR mutations [5].
Afatinib is an irreversible second-generation ErbB family blocker [6], which has been approved as a first-line treatment for NSCLC patients with EGFR exon 19 deletions or exon 21 L858R substitution mutations [7]. In 2013, afatinib was approved worldwide as a first-line treatment for patients with EGFR-mutant NSCLC [5, 8]. The LUX-Lung 3/6/7 trials revealed that afatinib had obvious effects in the treatment of advanced EGFR-mutant NSCLC [9-12] and might provide a better curative effect than first-generation EGFR-TKIs [13]. Moreover, a meta-analysis based on randomized controlled trials (RCTs) has shown that afatinib prolonged progression-free survival (PFS), increased overall 
survival (OS), and the overall response rate (ORR) [14]. However, whether afatinib is effective in particular subgroups remains controversial due to the RCT exclusion criteria $[14,15]$. Furthermore, the adverse effects of afatinib limit its clinical application [16]. Thus, a dose-adjustment strategy guided by tolerability can yield clinical benefits based on RCT data $[14,16]$, which need to be demonstrated through real-world evidence (RWE)-based data.

It is undeniable that the credibility of RCTs can be inferred from causality and is thus considered the gold standard of clinical research; however, RCTs may not reflect real-world practice due to strict inclusion criteria [17]. Patients in the real-world may differ in numerous characteristics from those in RCTs. RWE-based studies collect data of patients treated as a whole in clinical practice according to local government regulations, overcoming inherent limitations of RCTs, and can assess the efficacy and safety information of patients in the real world $[18,19]$. Therefore, it is crucial to confirm the efficacy and safety of afatinib by using RWE data. Additionally, a previous study based on real-world data proved that afatinib dose adjustment decreases the intensity and frequency of adverse drug reactions without affecting the efficacy [20]. Although RWE contributes to the evaluation of certain clinical influential factors, the impact of dose adjustment, brain metastasis, mutation type, and Eastern Cooperative Oncology Group Performance Status (ECOG-PS) on the efficacy and safety of afatinib remains elusive.

Accordingly, we performed a meta-analysis based on real-world afatinib data in advanced NSCLC with EGFR mutations. To the best of our knowledge, this is the first meta-analysis based on RWE to explore the efficacy and safety of afatinib in advanced NSCLC with EGFR mutations. In this study, related RWE from Embase, PubMed, and Cochrane Library databases were analyzed. Herein, we aimed to comprehensively analyze the efficacy and safety of afatinib in NSCLC patients with advanced EGFR mutations based on real-world evidence. Furthermore, we explored the impact of tolerability-guided dose adjustment, brain metastasis, mutation type, and ECOG-PS.

\section{Materials and Methods}

This meta-analysis was performed following the guidelines of the meta-analyses (PRISMA) statement [21].

2.1. Data Sources. Relevant studies were searched and identified in electronic databases, including Embase, PubMed, and Cochrane Library (updated to December 31, 2020). The main search keywords included "afatinib," "nonsmall cell lung cancer," NSCLC, "lung adenocarcinoma," and "adenosquamous carcinoma" The search was performed based on a combination of subject and free words. Additionally, a manual search of references in identified literature was performed to obtain additional information regarding the procedure. No language restrictions were applied to the current meta-analysis.
2.2. Inclusion and Exclusion Criteria. In the present study, the inclusion criteria employed were as follows: (i) the subjects were patients with advanced EGFR-mutated NSCLC diagnosed by histology and cytology; (ii) the study reported the efficacy (ORR, disease control rate (DCR), OS, PFS, and time to failure (TTF)) or safety (adverse reactions/ serious adverse reactions) of afatinib in the treatment of NSCLC; (iii) the study reported the difference in efficacy and safety based on different groups, including different afatinib doses (full dose $(40 \mathrm{mg} /$ day for 6 months or more) vs. nonfull dose $(<40 \mathrm{mg} /$ day for 6 months or $40 \mathrm{mg} /$ day for a reduction in the first 6 months)), mutation type (exon 19 deletion $v s$. uncommon, exon 21 L858R $v s$. uncommon, exon 19 deletion $v$ s. exon 21 L858R), ECOG-PS (0-1 vs. 2-4), and brain metastases; (iv) the research type was RWE.

The exclusion criteria were as follows: (i) nontreatment studies, including letters, reviews, and comments; (ii) the study lacked parameters required for quantitative analysis (including mean, sample size, standard deviation of the experimental group and the control group required for continuous variable meta-analysis) and could not be obtained through the conversion of other data in the study (enrolled as the qualitative description study); (iii) the study lacked baseline information such as gender, age, tumor classification, and ECOG-PS, reporting only the efficacy (PFS and TTF) of afatinib (enrolled as the qualitative description study); (iv) the research type was RCT. Furthermore, only one study (containing complete information) was extracted if more than one study was published using the same data.

2.3. Data Extraction and Quality Assessment. In the present study, two independent researchers participated in data extraction. The available data included first author name, year of publication, gender, sample size, history, country, age, stage, ORR, DCR, OS, PFS, TTF, and adverse reactions. The extraction tables were exchanged after both researchers had completed the above data extraction work. Any inconsistencies in extracted results were resolved through discussion.

2.4. Statistical Analysis. STATA software (version 11.0) was used for statistical analysis. The incidence rate (IR) and 95\% confidence interval (CI) were used as the effect size to evaluate the incidence of ORR, DCR, AEs, and SAEs in patients with NSCLC administering afatinib. The median ( $95 \% \mathrm{CI}$ ) was used to assess the months of PFS, OS, and TTF, while weighted mean difference (WMD) and 95\% CI were utilized as combined indices of effect quantity. The relative risk (RR) and 95\% CI were used as the combined indices of effect quantity to comprehensively evaluate differences in ORR, DCR, and incidence of adverse reactions between the two groups. Moreover, the risk of OS/PFS was analyzed using hazard risk (HR) and 95\% CI. If the study did not directly report the HR $(95 \% \mathrm{CI})$ of OS and PFS but reported the median survival and log-rank $P$-value in non-full dose $v s$. full dose of afatinib, the HR (95\% CI) could be converted using the method described before [22]. 
Cochran's $Q$ test and $I^{2}$ statistics were used to perform the heterogeneity analysis [23]. A random-effects model was used if heterogeneity was obtained $\left(P<0.05, I^{2}>50 \%\right)$; conversely, a fixed-effects model was employed. Moreover, to assess the effect of the above factors on the combined results of PFS, a subgroup analysis was conducted on PFS based on the timing of afatinib administration and data sources (direct reports in study or obtained by conversion). Furthermore, a sensitivity analysis was undertaken to assess the effect of combined results by analyzing the relevant studies individually. Finally, the Egger test [24] was used to analyze publication bias between the two groups.

\section{Results}

3.1. Literature Review and Characteristics of Included Studies. In total, 994, 990, and 281 studies were explored in the Embase, PubMed, and Cochrane Library databases, respectively (Figure 1). After eliminating duplicate studies, a total of 1525 studies were obtained, with 1479 studies further excluded as these failed to meet the inclusion criteria after assessing the abstract and title. Among the remaining 46 studies, 12 relevant studies were identified after reading the full text. Manual retrieval did not detect any study that could be included in the current analysis. Finally, a total of 25 studies [20, 25-33] with sufficient data were included (Table 1). A total of nine studies [34-41] were included in the current qualitative descriptive analysis (Table 2).

3.2. Study Characteristics. The 25 enrolled studies were published between 2014 and 2020. The study areas were primarily in Asia. Moreover, 19, 1, and 5 studies were enrolled in first-line, second-line, and mixed population groups, respectively.

3.3. Meta-Analysis for Efficacy and Safety of Afatinib Based on $R W E$. A total of 15 studies presented the outcomes of DCR (Figure 2(a)) and ORR (Figure 2(b)). Among these 15 studies, the heterogeneity was statistically significant $\left(I^{2}>\right.$ $50 \%, P<0.001$ ), and the combined result of DCR was $87.6 \%$ (95\% CI $[81.5 \%, 92.7 \%])$, and the difference between the first-line-only group $(90.8 \%$; 95\% CI [86.2\%, 94.6\%]) and second-line group (74.8\%; 95\% CI [52.5\%, 92.0\%]) was not significant $(P>0.05)$. The combined ORR was $58.9 \%(95 \%$ CI $[48.8 \%, 68.7 \%])$. The difference in ORR between the firstline-only group $(70.8 \%$; 95\% CI [67.2\%, 74.3\%]) and secondline group $(22.7 \%$; 95\% CI [16.8\%, 40.2\%]) was significant $(P<0.05)$.

Moreover, a total of 12 studies indicated the PFS outcomes of afatinib (Figure 3(a)), and the heterogeneity was statistically significant $\left(I^{2}>50 \%, P<0.05\right)$. The combined median PFS was 12.4 months (95\% CI [10.3, 14.5]). For PFS, the difference between the first-line-only group (13.6 months; 95\% CI $[12.4,14.7])$ and second-line group (6.1 months; 95\% CI $[2.3,9.9])$ was significant $(P<0.05)$. Furthermore, four studies reported TTF outcomes (Figure 3(b)). The heterogeneity was statistically significant $\left(I^{2}>50 \%\right.$, $P<0.05)$, and the combined median TTF was 15.4 months
(95\% CI [13.6-17.2]). The difference in TTF between the first-line-only group (15.7 months, 95\% CI $[13.4,18.0])$ and the mixed group (14.4 months, 95\% CI [11.5, 17.3]) was not significant $(P>0.05)$. Seven studies reported the OS outcomes in patients treated with afatinib (Figure 3(c)). The heterogeneity test among these seven studies was statistically significant $\left(I^{2}>50 \%, P<0.05\right)$. The combined median OS was 31.6 months (95\% CI [26.7-36.5]).

In total, 12 studies reported the incidence of diarrhea, mucositis, and skin rashes and 10 studies reported the total incidence of paronychia, including any grade (Figures 4(a)$4(\mathrm{~d})$ ) and Grade $\geq 3$ (Figures 5(a)-5(d)), respectively. The results revealed that the most common AEs were diarrhea, mucositis, skin rashes, and paronychia, with an incidence of 70.4\% (95\% CI [60.1\%, 79.8\%]) (Figure 4(a)), 36.5\% (95\% CI [29.5\%, 43.8\%]) (Figure 4(b)), 71.4\% (95\% CI [64.4\%, 77.9\%]) (Figure 4(c)), and 52.1\% (95\% CI [41.9\%, 62.3\%]) (Figure $4(\mathrm{~d})$ ), respectively. Meanwhile, the incidences of common SAEs (Grade $\geq 3$ ) for diarrhea, mucositis, skin rashes, and paronychia were 9.7\% (95\% CI [6.8\%, 13.1\%]) (Figure 5(a)), 2.1\% (95\% CI [1.0\%, 3.6\%]) (Figure 5(b)), 5.8\% (95\% CI [4.5\%, 7.2\%]) (Figure 5(c)), and 3.8\% (95\% CI [2.0\%, 6.2\%]) (Figure 5(d)), respectively.

Additionally, the total incidence of fatigue was $15.1 \%$ (95\% CI [4.1, 30.9], Supplementary Figure 1A) and the incidence of severe fatigue was $0.8 \%(95 \%$ CI $[0.0,2.2]$, Supplementary Figure 2A). The total and severe alanine aminotransferase (ALT) levels elevated were 8.3\% (95\% CI $[0.0 \%, 31.0 \%])$ (Supplementary Figure 1B) and 0.7\% (95\% CI $[0.0 \%, 2.3 \%])$ (Supplementary Figure 2B), respectively. The incidence of total and severe aspartate aminotransferase (AST) levels elevated was 6.2\% (95\% CI [0.0\%, 34.3\%]) (Supplementary Figure 1C) and 0.3\% (95\% CI [0.0\%, 1.5\%]) (Supplementary Figure 2C), respectively. The incidence of total and severe interstitial lung disease (ILD) was 1.1\% (95\% CI $[0.1 \%, 2.9 \%])$ (Supplementary Figure 1D) and 0.7\% (95\% CI $[0.0 \%, 2.3 \%])$ (Supplementary Figure 2D), respectively. Finally, except for skin rashes and fatigue, significant heterogeneity was observed among all studies that contained other indicators $\left(I^{2}>50 \%, P<0.05\right)$.

\subsection{Effect of Tolerability-Guided Afatinib Dose Adjustment.} Compared with the full dose of $40 \mathrm{mg} /$ day, 57.8\% (1917/ 3319) patients administered non-full dose of afatinib (Table 1). Seven studies (8 sets of research data) reported PFS between the non-full-dose and full-dose groups (Figure 6(a)). Among them, HRs (95\% CI) in four studies (five sets of data) were obtained by conversion. The heterogeneity for these data was not significant $\left(I^{2}<0.0 \%\right.$, $P>0.05)$. For the combined results, the HR was 1.2 (95\% CI $[0.9,1.5] ; P>0.05$ ) (Table 3, Figure 6(a)), which indicated that dose reduction does not impact the therapeutic efficacy in terms of PFS. The PFS of the non-full-dose group was 5.0-14.2 months, while the PFS in the full-dose group was 3.0-15.7 months. For the combined results, the WMD was -1.6 months $(95 \%$ CI $[-5.7,2.5])$ (Figure 6(b)). Two studies reported the difference in OS risk between the non-full-dose and full-dose groups (Figure 6(c)). The result of the 


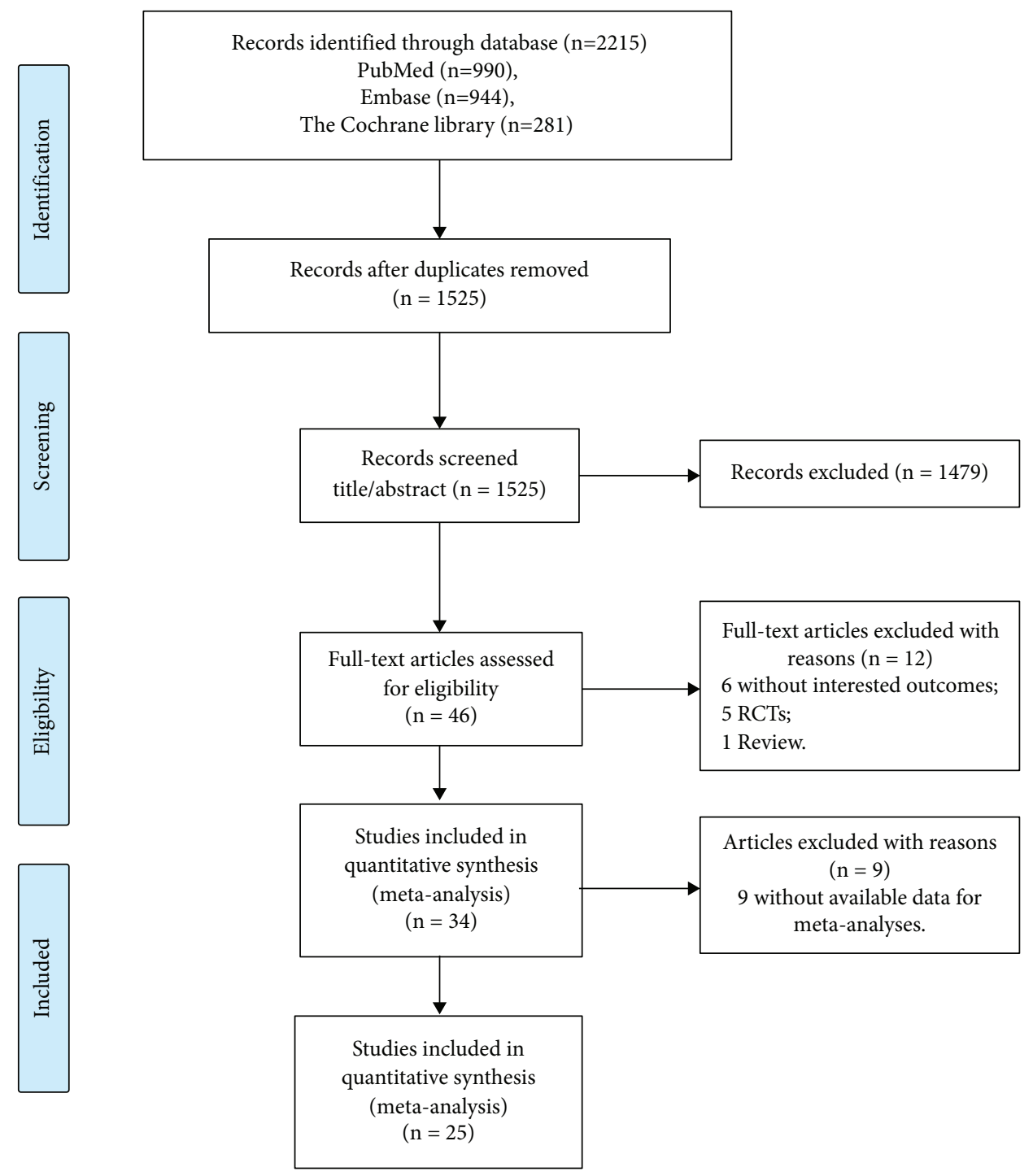

Figure 1: Flow diagram of the screening process for eligible studies.

heterogeneity test indicated that the heterogeneity between the two groups was not significant $\left(I^{2}<0.0 \%, P>0.05\right)$; thus, for the combined result, the HR was 1.03 (95\% CI $[0.69,1.5]$; $P>0.05)$, which indicated that dose reduction did not affect the therapeutic efficacy in terms of OS.

Three studies reported the difference between DCR and ORR based on different afatinib doses. The heterogeneity of DCR in these studies was significant $\left(I^{2}>50 \%, P>0.05\right)$. The non-full-dose group $(95.1 \%[88.0 \%, 99.4 \%])$ presented a DCR similar to the full-dose group [95.6\% (90.3, 99.1\%)]. For the combined results, the RR was 0.9 (95\% CI $[0.8,1.0]$; $P>0.05$ ) (Figure 7(a)). Moreover, the results of the heterogeneity test for ORR were $I^{2}<50 \%$ and $P>0.05$. The non-full-dose group $(67.8[56.8 \%, 77.9 \%])$ presented a significantly lower ORR than the full-dose group (78.5 $[66.7 \%, 88.4 \%])$. For the combined results, the RR was 0.8 (95\% CI $[0.7,0.9] ; P>0.05$ ) (Figure 7(b)). Although the difference in PFS and OS was not significant, the ORR was significantly higher in the full-dose group compared with the non-full-dose group.
Two studies reported differences in total AEs based on the full-dose and non-full-dose groups. Significant heterogeneity in the total AEs was observed in diarrhea and skin rashes $\left(I^{2}>50.0 \% ; P<0.05\right)$ but was not observed in mucositis and paronychia $\left(I^{2}>50.0 \%, \quad P<0.05\right)$ (Figure $8(\mathrm{a})$ ). The heterogeneity of diarrhea, skin rashes, and paronychia (Grade $\geq 3)$ was not significant $\left(I^{2}>50.0 \%\right.$, $P<0.05$ ) (Figure $8(\mathrm{~b})$ ). The incidence of total AEs and SAEs, including diarrhea, skin rash, mucositis, paronychia, dry skin, and pruritus, showed a decreased trend in the non-full-dose group (Table 4), which indicated the tolerability-guided dose adjustment alleviated afatinib-related adverse effects.

3.5. Effect of Subgroup on Efficacy and Safety of Afatinib. Two studies reported differences in the DCR and ORR in brain metastases. The DCRs in the without metastases group and the brain metastases group were $94.6 \%(91.3 \%, 97.3 \%)$ and $89.1 \%(82.2 \%, 94.6 \%)$, respectively. The ORRs in the 
TABle 1: Characteristics of 25 included studies in this meta-analysis.

\begin{tabular}{|c|c|c|c|c|c|c|c|c|c|c|}
\hline Study & Area & Afatinib & $\mathrm{N}$ & $\begin{array}{l}\text { Female, } \\
n(\%)\end{array}$ & $\begin{array}{l}\text { Age, years, } \\
\text { median } \\
\text { (range) }\end{array}$ & Mutations & $\begin{array}{l}\text { Clinical } \\
\text { stage }\end{array}$ & $\begin{array}{l}\text { ECOG- } \\
\text { PS, } 0-1 / \\
\geqq 2\end{array}$ & Histology & $\begin{array}{l}\text { Dose } \\
\text { reduce, } \\
\text { no/yes }\end{array}$ \\
\hline $\begin{array}{l}\text { Brat, K } \\
2020\end{array}$ & Czech & $\begin{array}{c}\text { First-line } \\
\text { only }\end{array}$ & 147 & $96(65.3)$ & $62.8 \pm 11.1$ & EGFR mutation & $\begin{array}{c}12 \text { IIIB, } 135 \\
\text { IV }\end{array}$ & $147 / 0$ & $145 \mathrm{AD}, 2$ other & NR/NR \\
\hline $\begin{array}{l}\text { Chen YH, } \\
2019\end{array}$ & Taiwan & $\begin{array}{l}\text { First-line } \\
\text { only }\end{array}$ & 23 & $15(65.2)$ & $42-81$ & $\begin{array}{l}17 \text { Del19, } 4 \\
\text { L858R, } 2 \\
\text { uncommon }\end{array}$ & 23 advanced & NR & NR & NR/NR \\
\hline $\begin{array}{l}\text { del Re M, } \\
2019\end{array}$ & Italy & $\begin{array}{l}\text { First-line } \\
\text { only }\end{array}$ & 41 & $20(48.8)$ & $70.5 \pm 11.3$ & $\begin{array}{c}34 \text { Del19, } 7 \\
\text { L858R }\end{array}$ & 2 IIIB, 39 IV & NR & NR & $26 / 15$ \\
\hline $\begin{array}{l}\text { Halmos B, } \\
2019\end{array}$ & Global & $\begin{array}{l}\text { First-line } \\
\text { only }\end{array}$ & 228 & $\begin{array}{c}138 \\
(60.5)\end{array}$ & $\begin{array}{c}67.0 \\
(32.0-90.0)\end{array}$ & $\begin{array}{c}178 \text { Del19, } 49 \\
\text { L858R }\end{array}$ & $\begin{array}{l}12 \text { IIIB, } 216 \\
\text { IV }\end{array}$ & $\begin{array}{l}\text { 192/27 (9 } \\
\text { missing) }\end{array}$ & $\begin{array}{c}226 \mathrm{AD}, 1 \mathrm{SCC} \\
1 \mathrm{NOS}\end{array}$ & $51 / 173$ \\
\hline $\begin{array}{l}\text { Ho GF, } \\
2019\end{array}$ & Malaysia & $\begin{array}{l}\text { First-line } \\
\text { only }\end{array}$ & 85 & $47(55.3)$ & $59.1 \pm 10.8$ & $\begin{array}{l}68 \text { Del19, } 11 \\
\text { L858R, } 6 \\
\text { uncommon }\end{array}$ & 4 IIIB, $81 \mathrm{IV}$ & $69 / 16$ & $82 \mathrm{AD}, 3 \mathrm{SCC}$ & $49 / 26$ \\
\hline $\begin{array}{l}\text { Hsieh YY, } \\
2019\end{array}$ & Taiwan & $\begin{array}{l}\text { First-line } \\
\text { only }\end{array}$ & 751 & $\begin{array}{c}424 \\
(56.5)\end{array}$ & $62.5 \pm 11.2$ & EGFR mutation & $\begin{array}{c}43 \text { IIIB, } 708 \\
\text { IV }\end{array}$ & $678 / 73$ & $\begin{array}{c}735 \mathrm{AD}, 16 \\
\text { other }\end{array}$ & NR/NR \\
\hline $\begin{array}{l}\text { Igawa S, } \\
2020\end{array}$ & Japan & $\begin{array}{l}\text { First-line } \\
\text { only }\end{array}$ & 48 & $27(56)$ & $67(35-85)$ & $\begin{array}{l}29 \text { Del19, } 19 \\
\text { L858R }\end{array}$ & $\begin{array}{l}36 \mathrm{IV}, 12 \\
\text { recurrence }\end{array}$ & $24 / 24$ & NR & $21 / 27$ \\
\hline $\begin{array}{l}\text { Ito } \mathrm{K} \text {, } \\
2020\end{array}$ & Japan & Mixed & 218 & $\begin{array}{c}102 \\
(46.8)\end{array}$ & $64.3(34-87)$ & $\begin{array}{l}141 \text { Del19, } 47 \\
\text { L858R, } 30 \\
\text { uncommon }\end{array}$ & $\begin{array}{c}39 \text { IA-IIIA, } \\
11 \text { IIIB, } 168 \\
\text { IV }\end{array}$ & $\begin{array}{c}203 / 12(3 \\
\text { missing) }\end{array}$ & $\begin{array}{c}208 \mathrm{AD}, 10 \\
\text { non-AD }\end{array}$ & NR/NR \\
\hline $\begin{array}{l}\text { Kan F, } \\
2014\end{array}$ & $\begin{array}{l}\text { United } \\
\text { Kingdom }\end{array}$ & $\begin{array}{l}\text { Second- } \\
\text { line or } \\
\text { more }\end{array}$ & 63 & $37(58.7)$ & $64(29-83)$ & $\begin{array}{l}15 \text { Del19, } 13 \\
\text { L858R, } 4 \\
\text { uncommon }\end{array}$ & $63 \mathrm{IV}$ & $\begin{array}{l}50 / 12(1 \\
\text { missing) }\end{array}$ & $\begin{array}{l}58 \mathrm{AD}, 1 \mathrm{SCC}, 2 \\
\text { NOS, } 1 \text { large } \\
\text { cell, } 1 \text { unknown }\end{array}$ & $33 / 31$ \\
\hline $\begin{array}{l}\text { Kim, Y } \\
2019\end{array}$ & Korea & $\begin{array}{l}\text { First-line } \\
\text { only }\end{array}$ & 165 & $80(48.5)$ & $57(30-79)$ & $\begin{array}{l}114 \text { Del19, } 37 \\
\text { L858R, } 14 \\
\text { uncommon }\end{array}$ & $165 \mathrm{IV}$ & $156 / 9$ & NR & $53 / 112$ \\
\hline $\begin{array}{l}\text { Lau SC, } \\
2019\end{array}$ & Canada & $\begin{array}{l}\text { First-line } \\
\text { only }\end{array}$ & 70 & $44(63)$ & $62(34-84)$ & $\begin{array}{l}\text { 41 Del19, } 20 \\
\text { L858R, } 9 \\
\text { uncommon }\end{array}$ & 70 advanced & $68 / 2$ & $66 \mathrm{AD}, 4$ other & NR/NR \\
\hline $\begin{array}{l}\text { Li YL, } \\
2019\end{array}$ & USA & $\begin{array}{l}\text { First-line } \\
\text { only }\end{array}$ & 87 & $62(71.3)$ & $69(62-81)$ & $\begin{array}{l}52 \text { Del19, } 35 \\
\text { L858R }\end{array}$ & 2 IIIB, 85 IV & $\begin{array}{l}31 / 10(46 \\
\text { missing) }\end{array}$ & $86 \mathrm{AD}, 1 \mathrm{SCC}$ & NR/NR \\
\hline $\begin{array}{l}\text { Liang SK, } \\
2017\end{array}$ & Taiwan & $\begin{array}{l}\text { First-line } \\
\text { only }\end{array}$ & 140 & $87(62.1)$ & $61(28-87)$ & $\begin{array}{l}81 \text { Del19, } 24 \\
\text { L858R, } 35 \\
\text { uncommon }\end{array}$ & $\begin{array}{l}4 \text { IIIB, } 136 \\
\text { IV }\end{array}$ & $129 / 11$ & $140 \mathrm{AD}$ & $81 / 59$ \\
\hline $\begin{array}{l}\text { Liang SK, } \\
2018\end{array}$ & Taiwan & $\begin{array}{c}\text { First-line } \\
\text { only }\end{array}$ & 259 & $\begin{array}{c}157 \\
(60.6)\end{array}$ & $62(28-87)$ & $\begin{array}{l}151 \text { Del19, } 53 \\
\text { L858R, } 55 \\
\text { uncommon }\end{array}$ & $\begin{array}{c}259 \\
\text { advanced }\end{array}$ & $240 / 19$ & $259 \mathrm{AD}$ & $139 / 120$ \\
\hline $\begin{array}{l}\text { Lin YT, } \\
2019\end{array}$ & Taiwan & $\begin{array}{l}\text { First-line } \\
\text { only }\end{array}$ & 99 & $61(61.6)$ & $\begin{array}{c}60(53-71) \\
\text { IQR }\end{array}$ & $\begin{array}{l}59 \text { Del19, } 23 \\
\text { L858R, } 13 \\
\text { uncommon }\end{array}$ & 99 advanced & $92 / 7$ & $\begin{array}{c}95 \mathrm{AD}, 4 \text { non- } \\
\mathrm{AD}\end{array}$ & NR/NR \\
\hline $\begin{array}{l}\text { Liu CY, } \\
2017\end{array}$ & Taiwan & $\begin{array}{l}\text { First-line } \\
\text { only }\end{array}$ & 146 & $78(53.4)$ & $63.2 \pm 11.3$ & $\begin{array}{l}73 \text { Del19, } 61 \\
\text { L858R, } 12 \\
\text { uncommon }\end{array}$ & $\begin{array}{c}16 \text { IIIB, } 130 \\
\text { IV }\end{array}$ & $123 / 23$ & $146 \mathrm{AD}$ & $79 / 67$ \\
\hline $\begin{array}{l}\text { Shen YC, } \\
2017\end{array}$ & Taiwan & $\begin{array}{l}\text { First-line } \\
\text { only }\end{array}$ & 24 & $15(62.5)$ & $59(33-86)$ & 24 uncommon & $1 \mathrm{IIIB}, 23 \mathrm{IV}$ & $19 / 5$ & $24 \mathrm{AD}$ & $24 / \mathrm{NR}$ \\
\hline $\begin{array}{l}\text { Sonehara } \\
\text { K, } 2019\end{array}$ & Japan & $\begin{array}{l}\text { First-line } \\
\text { only }\end{array}$ & 62 & $36(58.1)$ & $67(46-85)$ & $\begin{array}{c}42 \text { Del19, } 15 \\
\text { L858R, } 3 \\
\text { uncommon }\end{array}$ & $\begin{array}{c}5 \text { I-IIIA, } 5 \\
\text { IIIB, } 40 \mathrm{IV}, \\
13 \\
\text { recurrence }\end{array}$ & $57 / 5$ & $\begin{array}{c}61 \mathrm{AD}, 1 \\
\text { unclassified }\end{array}$ & $23 / 39$ \\
\hline $\begin{array}{l}\text { Tamura K, } \\
2019\end{array}$ & Japan & Mixed & 1602 & $\begin{array}{c}947 \\
(59.1)\end{array}$ & $67(34-90)$ & $\begin{array}{c}1020 \text { Del19, } 421 \\
\text { L858R, } 137 \\
\text { uncommon }\end{array}$ & $\begin{array}{l}94 \text { IIIB, } 1206 \\
\text { IV, other } \\
301,1 \\
\text { missing }\end{array}$ & $1381 / 221$ & $\begin{array}{l}1554 \text { AD, } 14 \\
\text { SCC, } 2 \text { NOS, } 1 \\
\text { large cell, } 32 \\
\text { others, } 1 \\
\text { unknown }\end{array}$ & $\begin{array}{l}580 / \\
1008\end{array}$ \\
\hline $\begin{array}{l}\text { Tan WL, } \\
2018\end{array}$ & Singapore & $\begin{array}{c}\text { First-line } \\
\text { only }\end{array}$ & 125 & $61(48.8)$ & $62(26-86)$ & $\begin{array}{l}87 \text { Del19, } 27 \\
\text { L858R, } 11 \\
\text { uncommon }\end{array}$ & $125 \mathrm{IV}$ & NR & $\begin{array}{c}121 \mathrm{AD}, 1 \mathrm{SCC} \\
3 \mathrm{NOS}\end{array}$ & $62 / 62$ \\
\hline
\end{tabular}


TABle 1: Continued.

\begin{tabular}{|c|c|c|c|c|c|c|c|c|c|c|}
\hline Study & Area & Afatinib & $\mathrm{N}$ & $\begin{array}{l}\text { Female, } \\
n(\%)\end{array}$ & $\begin{array}{l}\text { Age, years, } \\
\text { median } \\
\text { (range) }\end{array}$ & Mutations & $\begin{array}{l}\text { Clinical } \\
\text { stage }\end{array}$ & $\begin{array}{l}\text { ECOG- } \\
\text { PS, } 0-1 / \\
\geqq 2\end{array}$ & Histology & $\begin{array}{c}\text { Dose } \\
\text { reduce, } \\
\text { no/yes }\end{array}$ \\
\hline \multirow{2}{*}{$\begin{array}{l}\text { Tanaka H, } \\
2019\end{array}$} & \multirow{2}{*}{ Japan } & $\begin{array}{l}\text { First-line } \\
\text { only }\end{array}$ & 76 & $52(68.4)$ & $68(42-88)$ & $\begin{array}{l}46 \text { Del19, } 28 \\
\text { L858R, } 2 \\
\text { uncommon }\end{array}$ & $\begin{array}{c}9 \mathrm{IIIB}, 45 \mathrm{IV}, \\
22 \\
\text { recurrence }\end{array}$ & $67 / 9$ & $\begin{array}{c}74 \mathrm{AD}, 1 \mathrm{SCC}, 1 \\
\text { NOS }\end{array}$ & $18 / 58$ \\
\hline & & $\begin{array}{l}\text { Second- } \\
\text { line or } \\
\text { more }\end{array}$ & 52 & $41(78.9)$ & $65(39-90)$ & $\begin{array}{l}29 \text { Del19, } 21 \\
\text { L858R, } 2 \\
\text { uncommon }\end{array}$ & $\begin{array}{l}52 \\
\text { recurrence }\end{array}$ & $46 / 6$ & $51 \mathrm{AD}, 1 \mathrm{SCC}$ & NR/NR \\
\hline $\begin{array}{l}\text { Tu CY, } \\
2018\end{array}$ & Taiwan & $\begin{array}{l}\text { First-line } \\
\text { only }\end{array}$ & 104 & $65(62.5)$ & $\begin{array}{c}58<65 \\
\text { years/ } \\
46>65 \\
\text { years }\end{array}$ & $\begin{array}{l}58 \text { Del19, } 23 \\
\text { L858R, } 23 \\
\text { uncommon }\end{array}$ & $\begin{array}{l}3 \text { IIIB, } 101 \\
\text { IV }\end{array}$ & $93 / 11$ & $104 \mathrm{AD}$ & $67 / 31$ \\
\hline $\begin{array}{l}\text { Wada Y, } \\
2016\end{array}$ & Japan & Mixed & 73 & $46(63.0)$ & $69(42-85)$ & $\begin{array}{l}44 \text { Del19, } 20 \\
\text { L858R, } 5 \\
\text { uncommon, } 4 \\
\text { unknown }\end{array}$ & $\begin{array}{c}1 \text { IIIA, } 6 \\
\text { IIIB, } 44 \text { IV, } \\
22 \\
\text { recurrence }\end{array}$ & $56 / 17$ & $75 \mathrm{AD}$ & $36 / 37$ \\
\hline & & Mixed & 60 & $30(50.0)$ & $\begin{array}{c}58.1 \\
(36.3-82.7)\end{array}$ & $\begin{array}{l}26 \text { Del19, } 16 \\
\text { L858R, } 18 \\
\text { Uncommon }\end{array}$ & 60 advanced & $60 / 0$ & $60 \mathrm{AD}$ & $37 / 23$ \\
\hline $\begin{array}{l}\text { Wang S, } \\
2019\end{array}$ & China & $\begin{array}{c}\text { First-line } \\
\text { only }\end{array}$ & 39 & $23(59.0)$ & $\begin{array}{c}57.2 \\
(36.3-82.7)\end{array}$ & $\begin{array}{l}19 \text { Del19, } 7 \\
\text { L858R, } 13 \\
\text { uncommon }\end{array}$ & 39 advanced & $39 / 0$ & $39 \mathrm{AD}$ & NR/NR \\
\hline & & $\begin{array}{l}\text { Second- } \\
\text { line or } \\
\text { more }\end{array}$ & 21 & $7(33.3)$ & $\begin{array}{c}59.9 \\
(39.7-75.5)\end{array}$ & $\begin{array}{l}7 \text { Del19, } 9 \\
\text { L858R, } 5 \\
\text { uncommon }\end{array}$ & 21 advanced & $21 / 0$ & $21 \mathrm{AD}$ & NR/NR \\
\hline $\begin{array}{l}\text { Yang CJ, } \\
2017\end{array}$ & Taiwan & $\begin{array}{l}\text { First-line } \\
\text { only }\end{array}$ & 48 & $30(62.5)$ & $64.6 \pm 8.9$ & $\begin{array}{l}29 \text { Del19, } 19 \\
\text { L858R }\end{array}$ & $48 \mathrm{IV}$ & $38 / 10$ & $48 \mathrm{AD}$ & $19 / 29$ \\
\hline
\end{tabular}

NR, not reported; AD, adenocarcinoma; SCC, squamous cell carcinoma; NOS, not otherwise specified; ECOG-PS, Eastern Cooperative Oncology Group Performance Status; IQR, interquartile range.

TABLE 2: Characteristics of 9 studies in qualitative analysis.

\begin{tabular}{|c|c|c|c|c|c|c|c|c|c|}
\hline Study & Area & Afatinib & $\mathrm{N}$ & $\begin{array}{c}\text { Female, } n \\
(\%)\end{array}$ & $\begin{array}{c}\text { Age, years, } \\
\text { median (range) }\end{array}$ & Mutations & Clinical stage & $\begin{array}{c}\text { ECOG-PS, } \\
0-1 / \geq 2\end{array}$ & Histology \\
\hline $\begin{array}{l}\text { Fujiwara A } \\
2018\end{array}$ & Japan & $\begin{array}{l}\text { First-line } \\
\text { only }\end{array}$ & 28 & $19(67.9)$ & $68(37-82)$ & EGFR mutation & $\begin{array}{l}8 \text { IIIA, } 3 \text { IIIB, } 16 \\
\text { IV, } 1 \text { recurrence }\end{array}$ & NR & $\begin{array}{c}23 \mathrm{AD}, 3 \\
\text { SCC, } 2 \text { other }\end{array}$ \\
\hline $\begin{array}{l}\text { Hochmair } \\
\text { MJ, } 2018\end{array}$ & Global & $\begin{array}{l}\text { First-line } \\
\text { only }\end{array}$ & 204 & $110(53.9)$ & $60(30-86)$ & $\begin{array}{l}150 \text { Del19, } 53 \\
\text { L858R, } 1 \\
\text { uncommon }\end{array}$ & 197 IV, 7 missing & $\begin{array}{l}153 / 31(20 \\
\text { missing })\end{array}$ & NR \\
\hline $\begin{array}{l}\text { Jung HA, } \\
2020\end{array}$ & Korea & $\begin{array}{l}\text { First-line } \\
\text { only }\end{array}$ & 61 & NR & NR & $\begin{array}{c}\text { Uncommon EGFR } \\
\text { mutation }\end{array}$ & NR & NR & NR \\
\hline $\begin{array}{l}\text { Kanazu M, } \\
2020\end{array}$ & Japan & Mixed & 12 & NR & NR & $\begin{array}{c}\text { Uncommon EGFR } \\
\text { mutation }\end{array}$ & 12 advanced & NR & NR \\
\hline $\begin{array}{l}\text { Kuan FC, } \\
2017\end{array}$ & Taiwan & $\begin{array}{l}\text { First-line } \\
\text { only }\end{array}$ & 81 & $42(51.9)$ & $64(37-83)$ & 48 Del19, 33 L858R & 7 IIIB, 74 IV & $70 / 11$ & $81 \mathrm{AD}$ \\
\hline Lim, J 2019 & USA & $\begin{array}{c}\text { First-line } \\
\text { only }\end{array}$ & 550 & $355(64.5)$ & $63.3 \pm 11.4$ & EGFR mutation & NR & NR & NR \\
\hline Su VY, 2020 & Taiwan & $\begin{array}{l}\text { First-line } \\
\text { only }\end{array}$ & 99 & $52(52.5)$ & $64.1 \pm 10.8$ & $\begin{array}{l}53 \text { Del19, } 31 \\
\text { L858R, } 15 \\
\text { uncommon }\end{array}$ & 4 IIIB, 95 IV & $89 / 10$ & $\begin{array}{c}96 \mathrm{AD}, 3 \\
\text { non-AD }\end{array}$ \\
\hline $\begin{array}{l}\text { Wu SG, } 2020 \\
\text { (a) }\end{array}$ & Taiwan & $\begin{array}{l}\text { First-line } \\
\text { only }\end{array}$ & 36 & $27(75)$ & $\begin{array}{c}68.7 \\
(43.0-86.1)\end{array}$ & 36 uncommon & $\begin{array}{c}32 \mathrm{IV}, 4 \\
\text { recurrence }\end{array}$ & $32 / 4$ & $36 \mathrm{AD}$ \\
\hline $\begin{array}{l}\text { Wu SG, } 2020 \\
\text { (b) }\end{array}$ & Taiwan & $\begin{array}{l}\text { First-line } \\
\text { only }\end{array}$ & 91 & $44(48.4)$ & $63(37-83)$ & $\begin{array}{c}59 \text { Del19, } 21 \\
\text { L858R, } 11 \\
\text { uncommon }\end{array}$ & $\begin{array}{l}83 \text { advanced, } 8 \\
\text { recurrence }\end{array}$ & NR & $91 \mathrm{AD}$ \\
\hline
\end{tabular}

Notes: NR, not reported; AD, adenocarcinoma; SCC, squamous cell carcinoma; ECOG-PS, Eastern Cooperative Oncology Group Performance Status.

without metastases group and the brain metastases group were $74.9 \%(95 \%$ CI $[69.1 \%, 80.2 \%])$ and $61.8 \%(95 \%$ CI $[52.2 \%, 71.0 \%])$, respectively. The differences in DCR (RR:
1.0, 95\% CI $[0.9,1.1] ; P>0.05)$ and ORR (RR: $2.0,95 \% \mathrm{CI}$ $[0.5,7.6] ; P>0.05$ ) (Figures $9(\mathrm{a})$ and $9(\mathrm{~b})$ ) were not significant. 


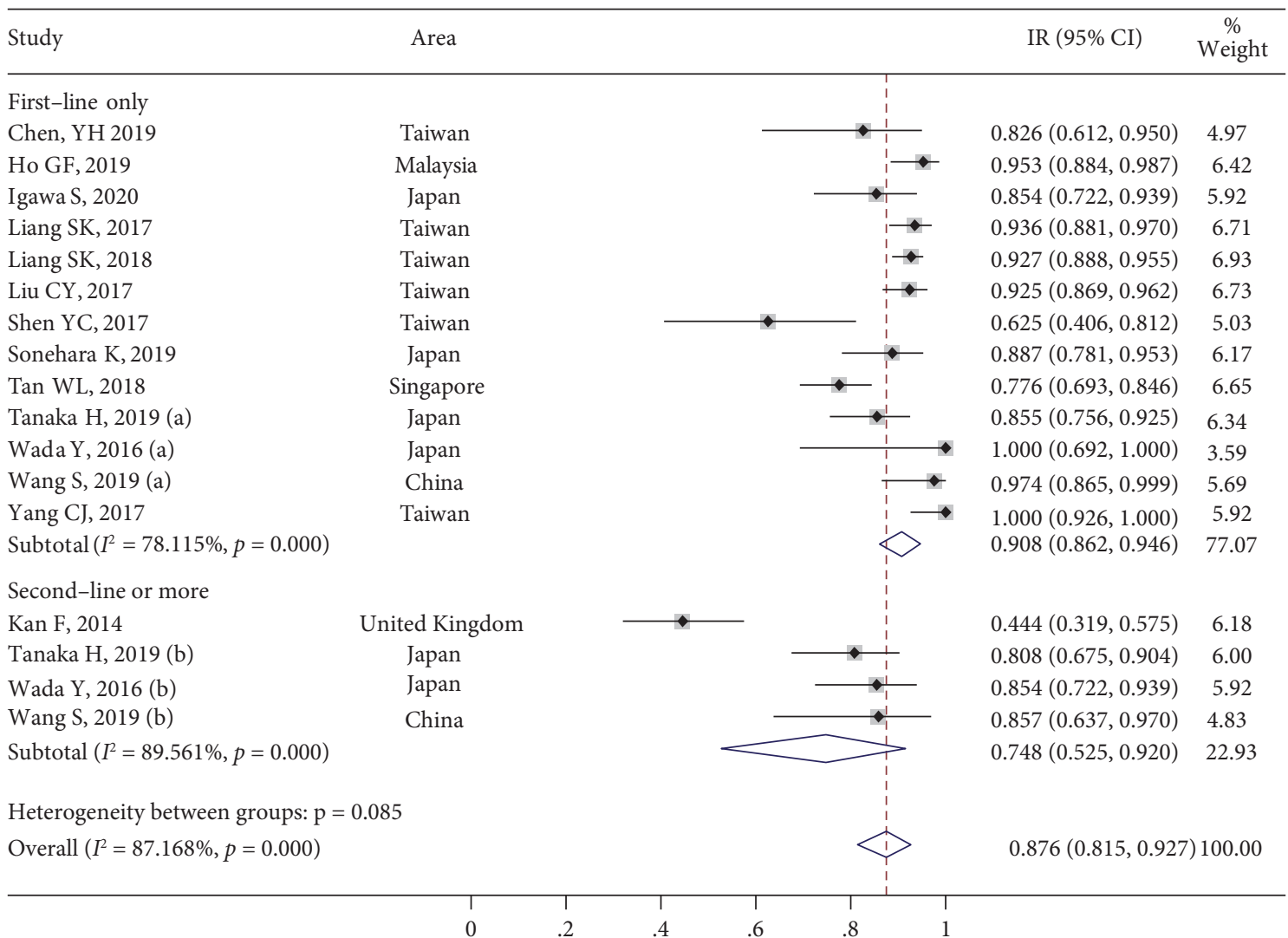

(a)

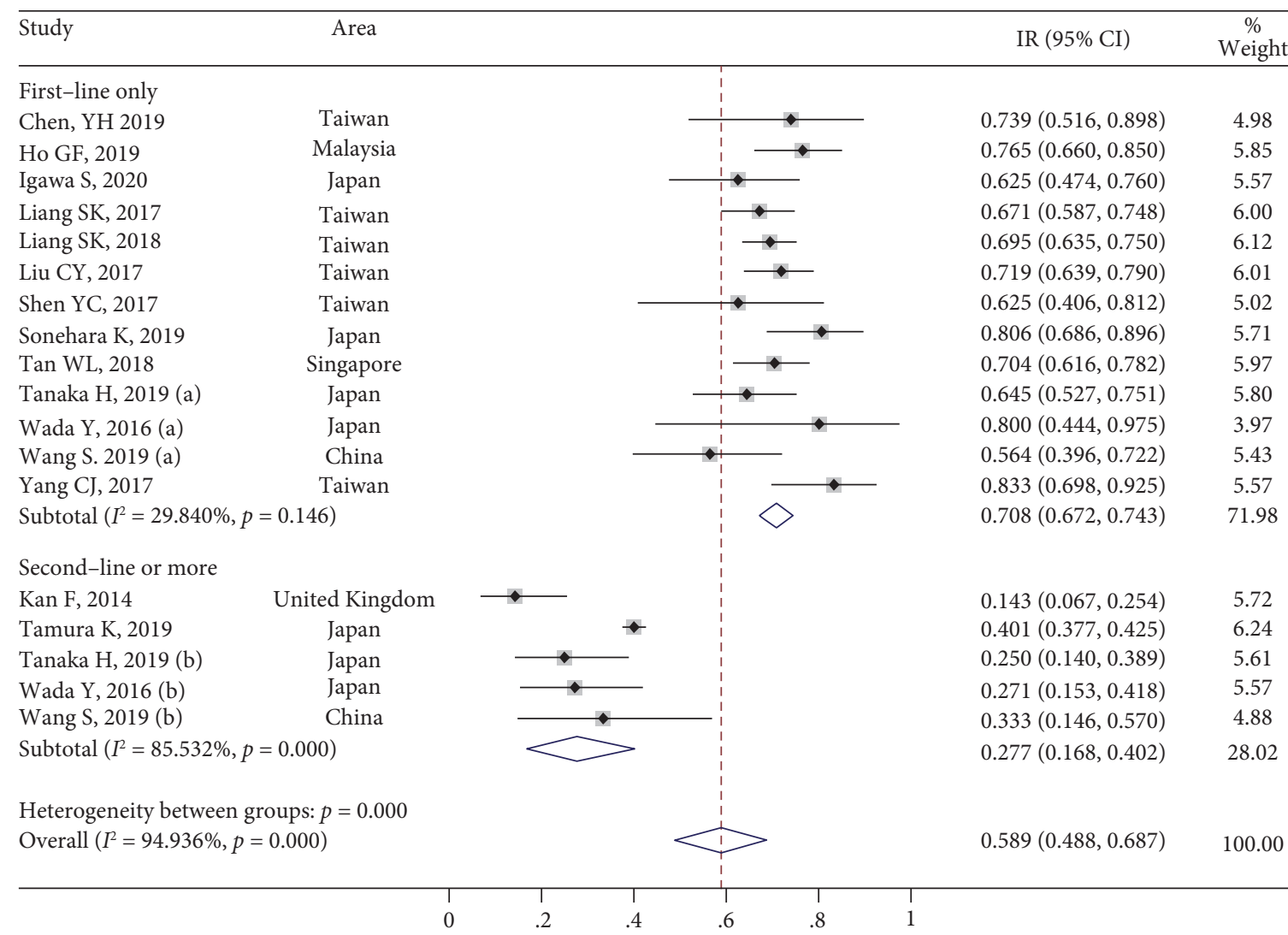

(b)

FIGURE 2: The meta-analysis results of afatinib efficacy and safety in advanced NSCLC with EGFR mutation: (a) the outcome of disease control rates (DCRs) and (b) the outcome of objective response rates (ORRs). 


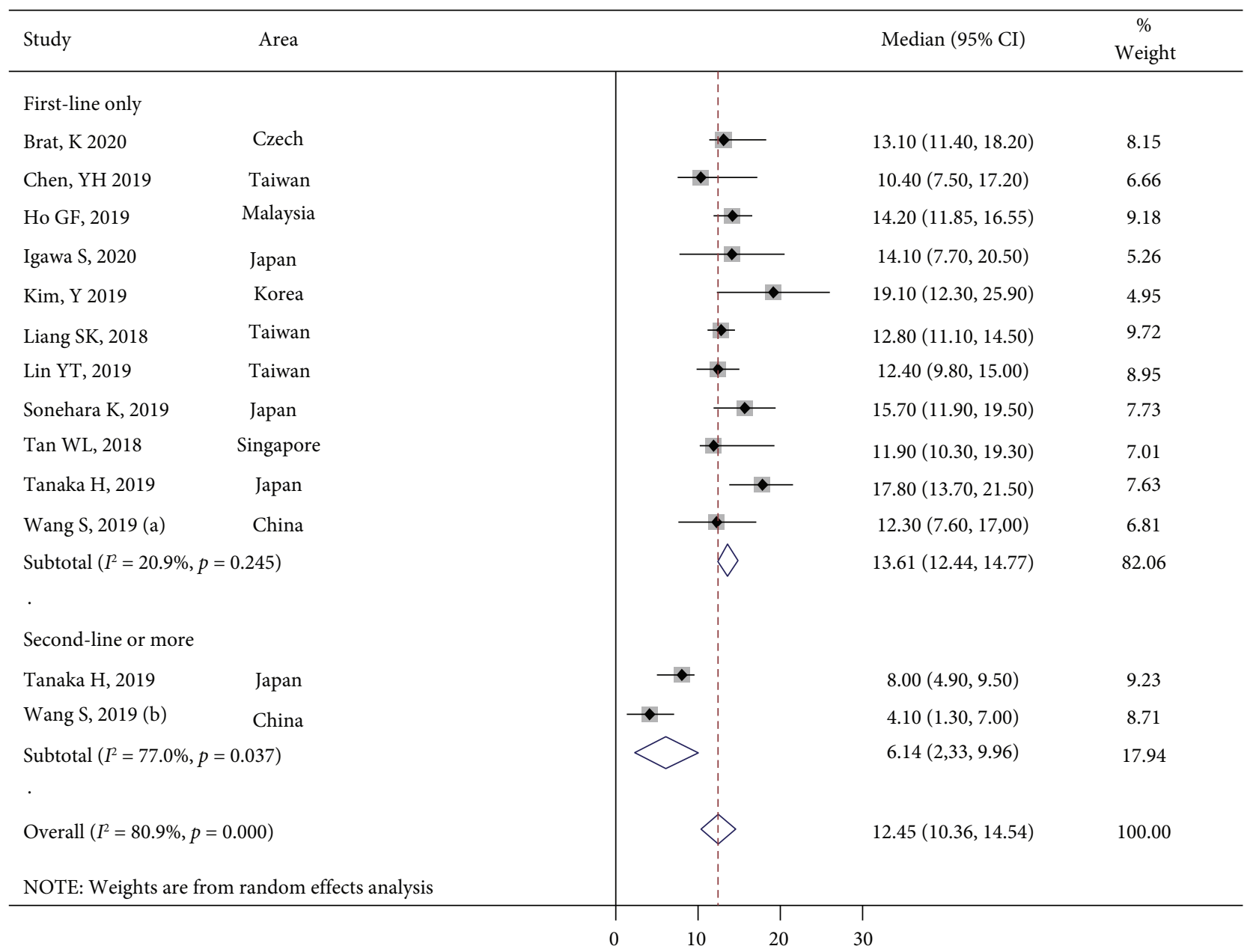

(a)

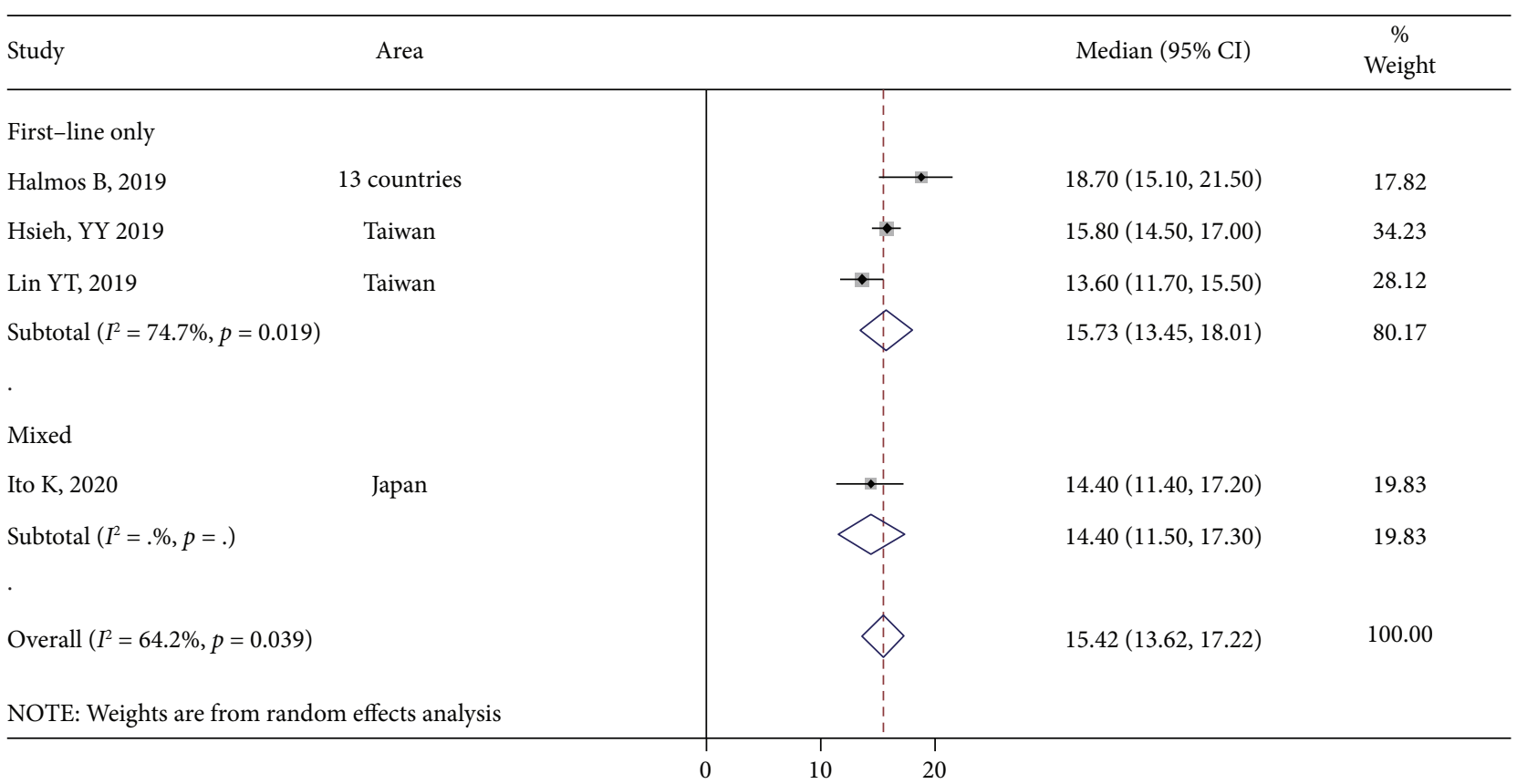

(b)

FIgUre 3: Continued. 


\begin{tabular}{|c|c|c|c|c|c|}
\hline Study & Area & Afatinib & & Median $(95 \%$ CI $)$ & $\begin{array}{c}\% \\
\text { Weight }\end{array}$ \\
\hline Brat, K 2020 & Czech & First-line only & $\rightarrow \frac{1}{1}$ & $29.30(20.90,38.60)$ & 14.68 \\
\hline Chen, YH 2019 & Taiwan & First-line only & 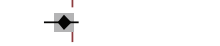 & $29.60(24.80,33.00)$ & 23.59 \\
\hline Ho GF, 2019 & Malaysia & First-line only & $\longrightarrow$ & $28.90(19.82,37.99)$ & 14.31 \\
\hline Igawa S, 2020 & Japan & First-line only & - & $48.30(31.40,65.20)$ & 6.46 \\
\hline Lau SC, 2019 & Canada & First-line only & & $39.00(25.60,48.80)$ & 10.92 \\
\hline Li YL, 2019 & USA & First-line only & $\rightarrow$ & $20.70(16.20,35.10)$ & 13.76 \\
\hline Lin YT, 2019 & Taiwan & First-line only & & $37.00(25.10,40.90)$ & 16.27 \\
\hline \multicolumn{3}{|c|}{ Overall $\left(I^{2}=55.2 \%, p=0.037\right)$} & & $31.67(26.78,36.56)$ & 100.00 \\
\hline \multicolumn{4}{|c|}{ NOTE: Weights are from random effects analysis } & & \\
\hline
\end{tabular}

(c)

FIGURE 3: The meta-analysis results of afatinib efficacy and safety in advanced NSCLC with EGFR mutation: (a) the outcome of progressionfree survival (PFS); (b) the outcome for overall survival (OS); and (c) the outcome for time to failure (TTF).

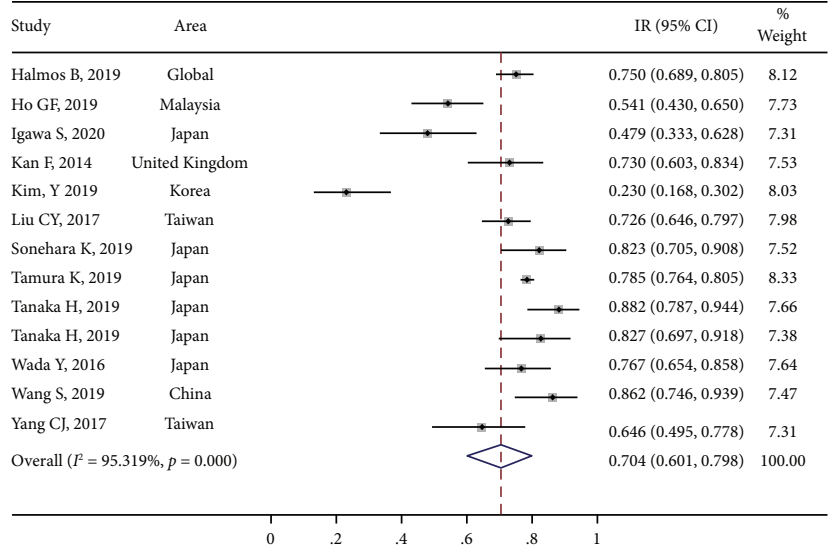

(a)

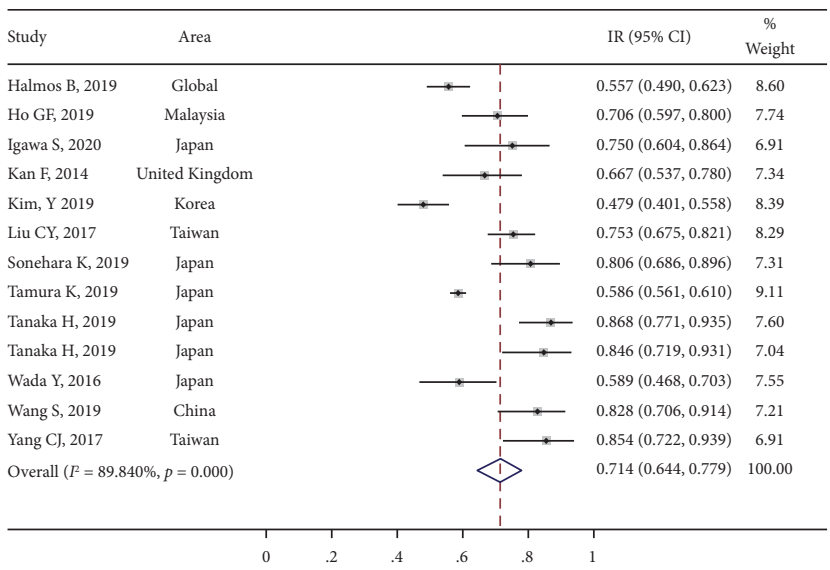

(c)

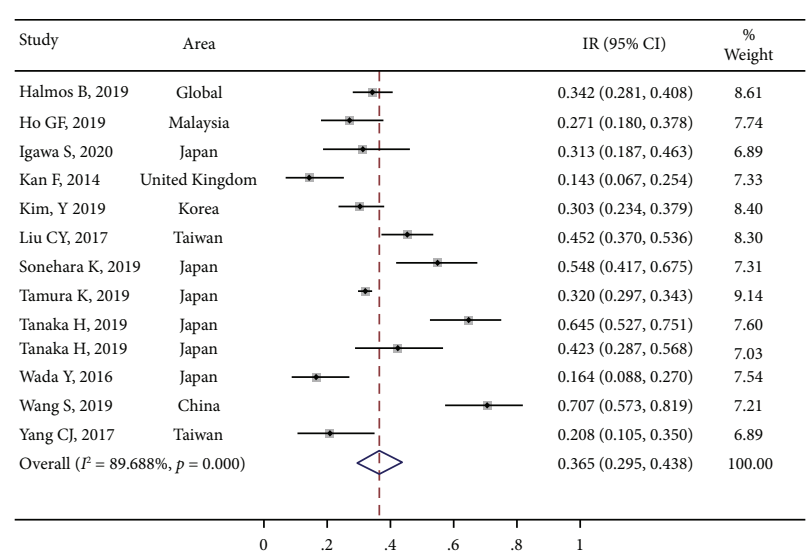

(b)

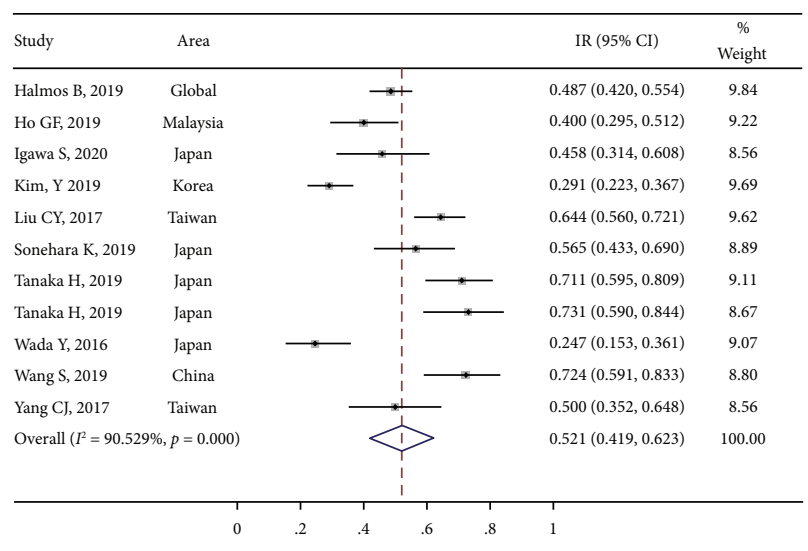

(d)

FIGURE 4: The meta-analysis results for various incidence rates after afatinib treatment in advanced NSCLC with EGFR mutation: (a) the incidence rate of diarrhea; (b) the incidence rate of mucositis; (c) the incidence rate of skin rashes; and (d) the incidence rate of paronychia. 


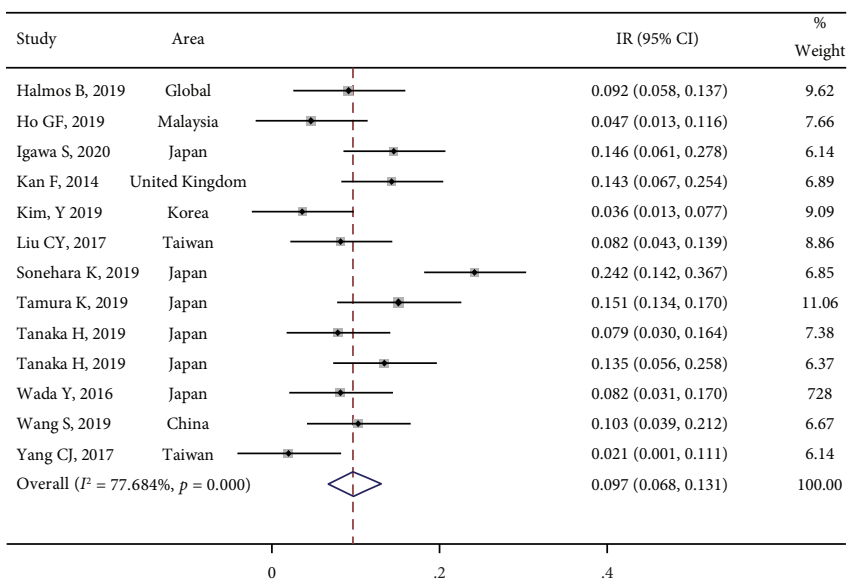

(a)

\begin{tabular}{|c|c|c|c|c|}
\hline Study & \multicolumn{2}{|l|}{ Area } & IR $(95 \% \mathrm{Cl})$ & $\begin{array}{c}\% \\
\text { Weight }\end{array}$ \\
\hline Halmos B, 2019 & Global & + & $0.083(0.051,0.127)$ & 12.31 \\
\hline Ho GF, 2019 & Malaysia & i & $0.059(0.019,0.132)$ & 5.85 \\
\hline Igawa S, 2020 & Japan & $\frac{1}{1}$ & $0.083(0.023,0.200)$ & 3.56 \\
\hline Kan F, 2014 & United Kingdom & 1 & $0.079(0.026,0.176)$ & 4.53 \\
\hline Kim, Y 2019 & Korea & $\rightarrow-1$ & $0.018(0.004,0.052)$ & 9.83 \\
\hline Liu CY, 2017 & Taiwan & $\frac{1}{4}$ & $0.055(0.024,0.105)$ & 8.98 \\
\hline Sonehara K, 2019 & Japan & $\frac{1}{1}$ & $0.081(0.027,0.178)$ & 4.47 \\
\hline Tamura K, 2019 & Japan & $\div$ & $0.058(0.047,0.071)$ & 28.41 \\
\hline Tanaka H, 2019 & Japan & 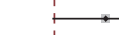 & $0.118(0.056,0.213)$ & 5.32 \\
\hline Tanaka H, 2019 & Japan & 1 & $0.038(0.005,0,132)$ & 3.83 \\
\hline Wada Y, 2016 & Japan & $\stackrel{1}{1}$ & $0.068(0.023,0.153)$ & 5.14 \\
\hline Wang S, 2019 & China & $\rightarrow-1$ & $0.034(0.004,0.119)$ & 4.21 \\
\hline Yang CJ, 2017 & Taiwan & 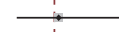 & $0.063(0.013,0.172)$ & 3.56 \\
\hline \multicolumn{2}{|c|}{ Overall $\left(I^{2}=24.285 \%, p=0.198\right)$} & $\begin{array}{l}\text { (1) } \\
1 \\
1\end{array}$ & $0.058(0.045,0.072)$ & 100.00 \\
\hline
\end{tabular}

(c)

\begin{tabular}{|c|c|c|c|c|}
\hline Study & Area & & $\operatorname{IR}(95 \% \mathrm{CI})$ & $\begin{array}{c}\% \\
\text { Weight }\end{array}$ \\
\hline Halmos B, 2019 & Global & $\rightarrow$ & $0.057(0.031,0.096)$ & 10.89 \\
\hline Ho GF, 2019 & Malaysia & + & $0.024(0.003,0.082)$ & 7.35 \\
\hline Igawa S, 2020 & Japan & + & $0.021(0.001,0.111)$ & 5.27 \\
\hline Kan F, 2014 & United Kingdom & $\frac{1}{1}$ & $0.016(0.000,0.085)$ & 6.23 \\
\hline Kim, Y 2019 & Korea & $\rightarrow$ & $0.000(0.000,0.022)$ & 9.81 \\
\hline Liu CY, 2017 & Taiwan & $\rightarrow$ & $0.048(0.019,0.096)$ & 9.38 \\
\hline Sonehara K, 2019 & Japan & 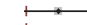 & $0.065(0.018,0.157)$ & 6.17 \\
\hline Tamura K, 2019 & Japan & $1 *$ & $0.039(0.030,0.049)$ & 14.44 \\
\hline Tanaka H, 2019 & Japan & $\rightarrow$ & $0.026(0.003,0.092)$ & 6.93 \\
\hline Tanaka H, 2019 & Japan & $\frac{T}{1}$ & $0.000(0.000,0.068)$ & 5.54 \\
\hline Wada Y, 2016 & Japan & th & $0.027(0.003,0.095)$ & 6.78 \\
\hline Wang S, 2019 & China & 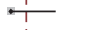 & $0.000(0.000,0.062)$ & 5.93 \\
\hline Yang CJ, 2017 & Taiwan & +1 & $0.000(0.000,0.074)$ & 5.27 \\
\hline \multirow{2}{*}{\multicolumn{2}{|c|}{ Overall $\left(I^{2}=60.806 \%, p=0.002\right)$}} & $\begin{array}{l}\Delta \\
1 \\
1\end{array}$ & $0.021(0.010,0.036)$ & 100.00 \\
\hline & & 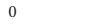 & .4 & \\
\hline
\end{tabular}

(b)

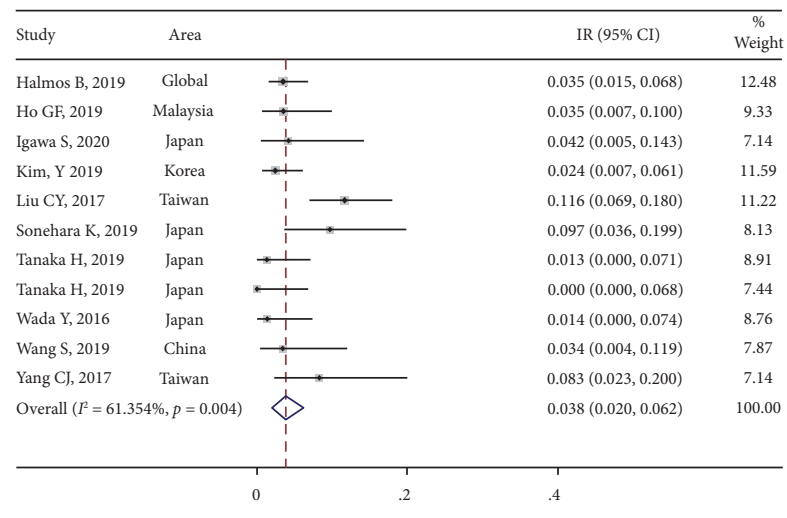

(d)

FIGURE 5: The meta-analysis results for serious adverse reaction incidence rates after afatinib treatment in advanced NSCLC with EGFR mutation: (a) the adverse reaction incidence rate of diarrhea; (b) the adverse reaction incidence rate of mucositis; (c) the adverse reaction incidence rate of skin rashes; and (d) the adverse reaction incidence rate of paronychia.

Furthermore, two studies reported differences in DCR/ ORR between mutation types. The DCRs in the exon 19 deletion group, exon 21 L858R group, and uncommon group were $94.0 \%$ (95\% CI [89.4, 97.4\%]), 92.7\% (95\% CI [80.5\%, $99.7 \%]$ ), and $92.8 \%$ (95\% CI [97.2\%, 100.0\%]), respectively. The differences in DCR in the exon 19 deletion $v s$. uncommon group (RR: $1.1,95 \%$ CI $[0.9,1.2] ; P>0.05)$ and exon 21 L858R vs. uncommon group (RR: 1.1, 95\% CI [0.9, 1.3]; $P>0.05$ ) were not significant (Figure 9(c)). The ORRs in the exon 19 deletion group, exon 21 L858R group, and uncommon group were $71.9 \%$ (95\% CI [64.3\%, 78.9\%]), $69.1 \%$ (95\% CI $[55.2 \%, 84.0 \%])$, and $59.9 \%$ (95\% CI $[41.3 \%$, $77.4 \%]$ ), respectively. The differences in ORR in the exon 19 deletion $v s$. uncommon group (RR: 1.3 , 95\% CI $[0.9,1.9]$, $P>0.05$ ) and exon 21 L858R vs. uncommon group (RR: 1.3, $95 \%$ CI $[0.9,2.0] ; \quad P>0.05)$ were not significant (Figure 9(d)).

Moreover, two studies reported PFS in patients with brain metastases based on the full-dose and non-full-dose groups (Supplementary Figure 3A). For the combined results, the HR was 2.4 (95\% CI $[0.9,5.9] ; P>0.05)$. Furthermore, the combined results showed that the PFS in the exon 19 deletion group was undoubtedly higher than that in the uncommon mutation group (HR: $0.2,95 \%$ CI $[0.1,0.4]$; $P<0.05)$, and PFS in patients without brain metastasis was significantly lower than that in patients with brain metastasis (HR: $0.5 ; 95 \%$ CI $[0.4,0.8] ; \quad P<0.05$ ) (Supplementary Figure $3 \mathrm{~B})$. No significant difference was observed in ECOGPS (0-1) vs. ECOG-PS ( $\geq 2)$ (HR: 0.3, 95\% CI [0.1, 1.4]; $P>0.05)$ (Supplementary Figure $3 \mathrm{~B})$. However, the heterogeneity between the two studies was significant $\left(I^{2}=59.4 \%\right)$ (Figure 6(c)).

3.6. Sensitivity Analysis and Publication Bias. Publication bias and sensitivity analyses were performed on the study outcomes. The sensitivity analysis results revealed that none of the included studies had a noticeable influence on the combined result of PFS between the full-dose and the nonfull-dose groups. The combined results of PFS ranged from HR: $1.1(95 \%$ CI $[0.8,1.4])$ to HR: 1.3 (95\% CI $[0.9,1.7])$ $(P>0.05)$ following removal of any single study. Furthermore, the Egger test showed that publication bias in the current meta-analysis was not significant $(P>0.05)$. 


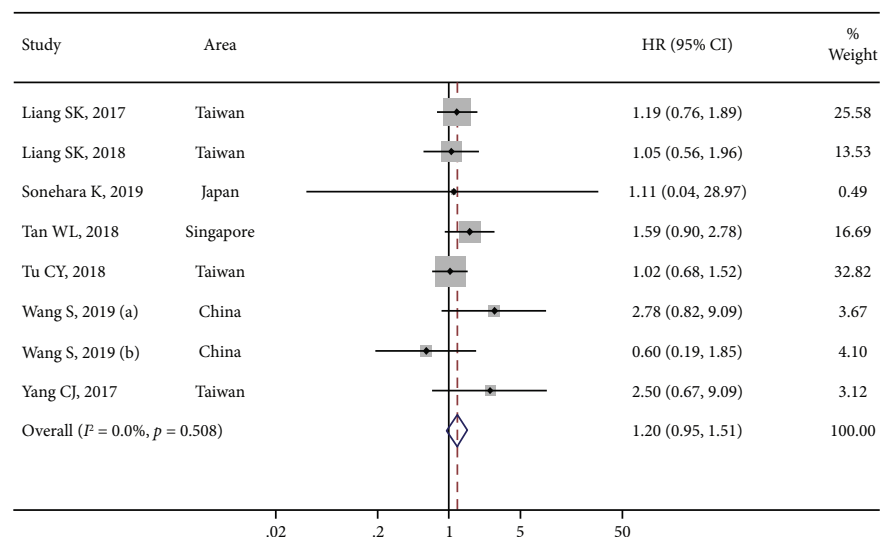

(a)

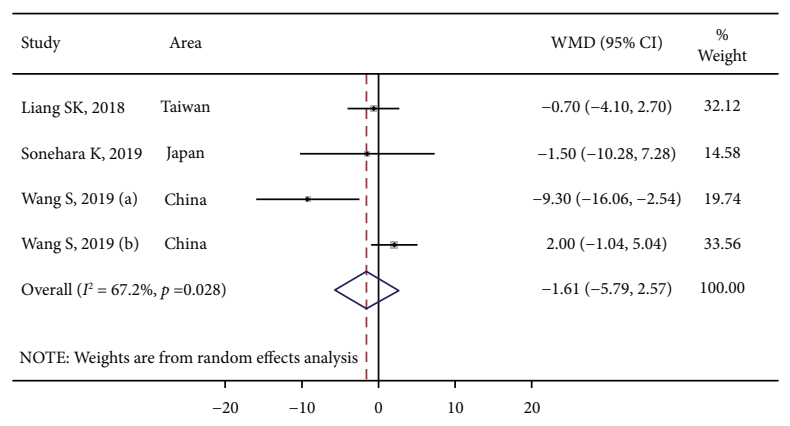

(b)

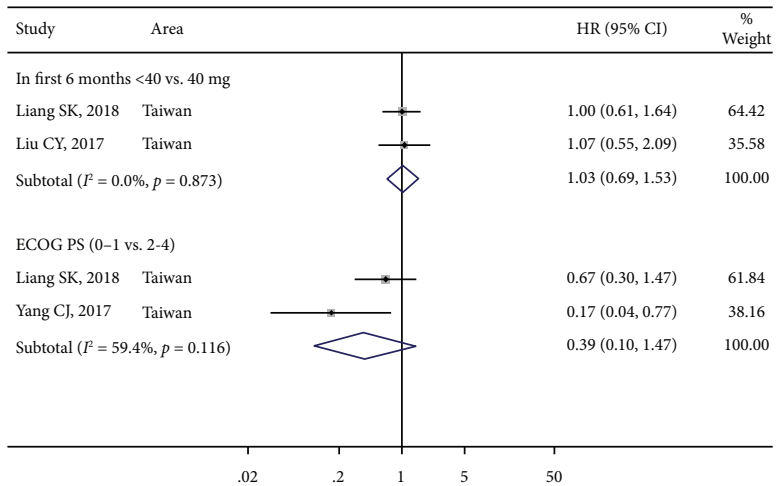

(c)

FIGURE 6: The meta-analysis results for progression-free survival (PFS) among groups after afatinib treatment in advanced NSCLC with EGFR mutation: (a) PFS between non-full-dose group and full-dose group; (b) PFS between the full-dose group and non-full-dose group; and (c) the OS between non-full-dose group and full-dose group in advanced NSCLC patients with epidermal growth factor receptor (EGFR) positive mutations after afatinib treatment.

TABLE 3: Subgroup analysis based on afatinib medication timing and data sources.

\begin{tabular}{lcccc}
\hline Group & No. of studies & HR $(95 \% \mathrm{CI})$ & $P_{A}$ & \multicolumn{2}{c}{ Heterogeneity test } \\
\hline PFS & 8 & $1.20(0.95,1.51)$ & 0.124 & 0.508 \\
Management of afatinib & & $1.23(0.98,1.56)$ & 0.080 & 0.0 \\
$\quad$ First-line & 7 & $0.60(0.19,1.87)$ & 0.379 & 0.570 \\
$\quad$ Second-line & 1 & & & - \\
Calculated HR (95\%CI) & 5 & $1.06(0.77,1.44)$ & 0.728 & 0.483 \\
$\quad$ Yes & 3 & $1.39(0.99,1.96)$ & 0.057 & 0.486 \\
$\quad$ No & & & 0.0 \\
\hline
\end{tabular}

Notes: $P_{A}: P$ value for the test of association; $P_{H}: P$ value for the test of heterogeneity.

\section{Discussion}

Based on RCT data, a meta-analysis has revealed that in the first-line treatment of EGFR-mutated NSCLC, there is no conclusive evidence that afatinib is more effective than gefitinib or erlotinib [15]. Meanwhile, Wang et al. have performed a meta-analysis of RCTs in advanced NSCLC to assess the safety and efficacy of afatinib when compared with chemotherapy and first-generation EGFR-TKIs. Their results revealed that compared with control groups, afatinib treatment apparently increased ORR (RR: 1.8, 95\% CI [1.1-2.9]) and improved PFS (HR: 0.5; 95\% CI) and improved OS (HR: 0.9, 95\% CI [0.8-0.9]). In terms of safety, the incidence of adverse events (Grade $\geq 3$ ) was as follows: diarrhea (11.8\%) (RR: 8.9, 95\% CI [5.3-14.9]), stomatitis (4.8\%) (RR: 6.4, 95\% CI [1.2-32.7]), and skin rash (10.7\%) (RR: 7.3, 95\% CI [1.5-34.1]) [14]. In this RWEbased meta-analysis, the results confirmed that the afatinib was with ORR 58.9\% (48.8, 68.7), PFS 12.4 months (10.3, 14.5), TTF 15.4 months $(13.6,17.2)$, and OS 31.6 months (26.7, 36.5), which is consistent with RCT results. The incidences of severe adverse events (Grade $\geq 3$, SAEs) for diarrhea, skin rashes, paronychia, and mucositis were $9.7 \%$ (6.8\%, 13.1\%), 5.8\% (4.5\%, 7.2\%), 3.8\% (2.0\%, 6.2\%), and 


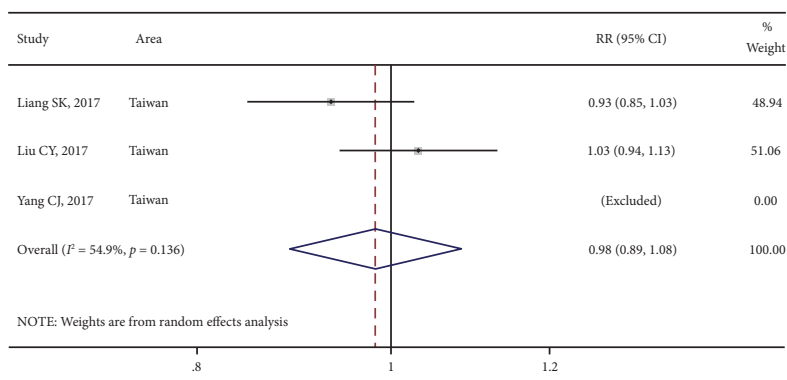

(a)

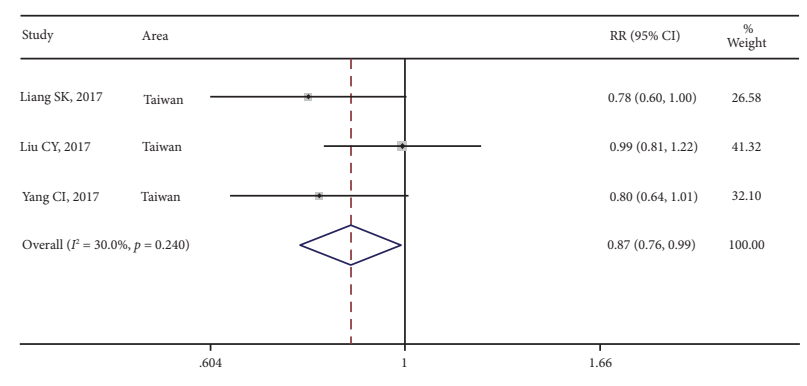

(b)

FIgURE 7: The meta-analysis results for disease control rates (DCRs) and objective response rates (ORRs) in different dose groups after afatinib treatment in advanced NSCLC with EGFR mutation: (a) comparison of DCRs in different dose groups and (b) comparison of ORRs in different dose groups.

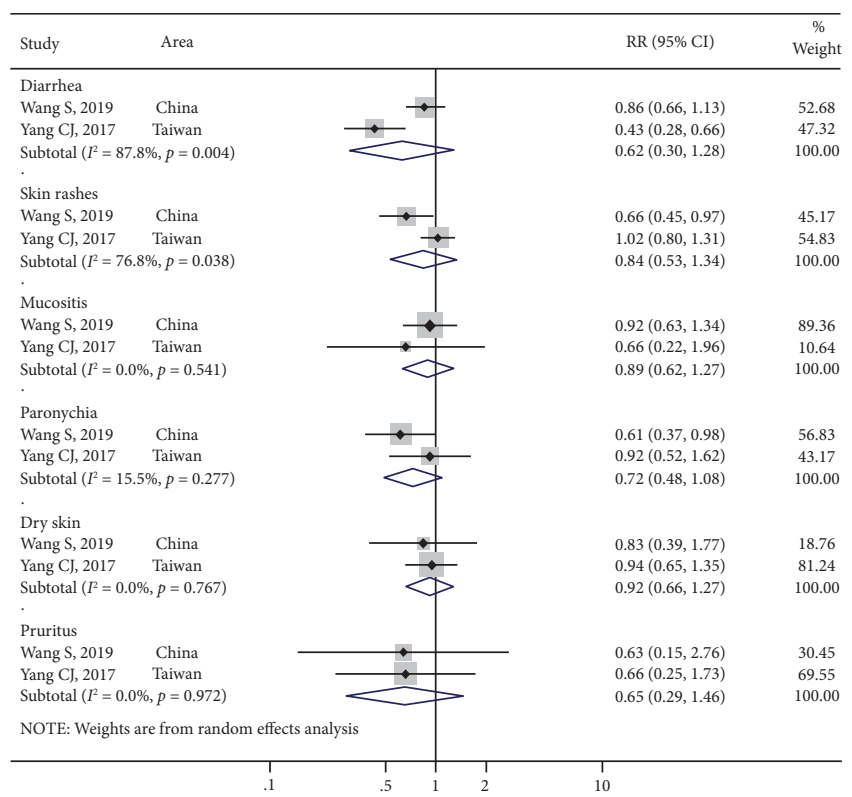

(a)

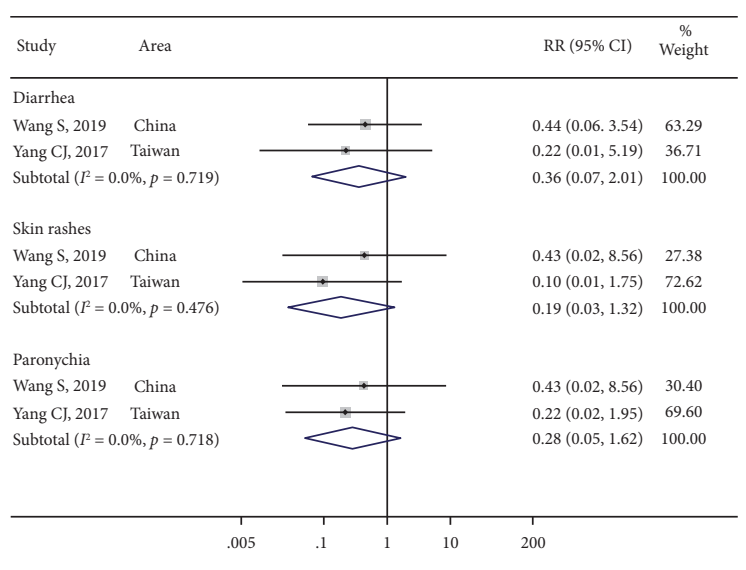

(b)

Figure 8: The meta-analysis results for total adverse events and severe adverse events in different dose groups after afatinib treatment in advanced NSCLC with EGFR mutation: (a) comparison of total adverse events in different dose groups and (b) comparison of severe adverse events in different dose groups.

TABle 4: Total AEs (or SAEs) based on different afatinib dose (non-full dose vs. full-dose).

\begin{tabular}{lcccc}
\hline \multirow{2}{*}{ AEs (or SAEs) } & \multicolumn{2}{c}{ Non-full dose } & \multicolumn{2}{c}{ Full dose } \\
& Total & Grade $\geq 3$ & $94.7(86.8,99.5) \%$ & $9.8(3.0,19.3) \%$ \\
\hline Diarrhea & $55.8(41.1,70.0) \%$ & $1.0(0.0,7.4) \%$ & $90.3(80.9,97.1) \%$ & $7.7(1.7,16.5) \%$ \\
Skin rashes & $77.7(64.2,88.9) \%$ & $0.0(0.0,4.0) \%$ & $58.0(44.9,70.6) \%$ & $0.0(0.0,3.0) \%$ \\
Mucositis & $34.7(21.4,49.2) \%$ & $0.0(0.0,4.0) \%$ & $74.0(61.7,84.7) \%$ & $7.7(1.7,16.5) \%$ \\
Paronychia & $48.9(34.4,63.5) \%$ & $1.5(0.0,8.4) \%$ & $51.0(38.0,64.0) \%$ & $0.0(0.0,3.0) \%$ \\
Dry skin & $55.6(40.9,69.8) \%$ & $0.0(0.0,4.0) \%$ & $21.5(11.6,33.3) \%$ & $0.0(0.0,3.0) \%$ \\
Pruritus & $16.7(6.9,29.3) \%$ & $0.0(0.0,4.0) \%$ & &
\end{tabular}

AEs, adverse events; SAEs, severe adverse events.

$2.1 \%(1.0 \%, 3.6 \%)$, respectively. Furthermore, in the present study, the efficacy of afatinib in the first-line-only group was significantly superior to that in the second-line treatment. Therefore, the efficacy and safety of afatinib has been confirmed by RWE.
The efficacy of tolerability-guided dose adjustment remains controversial. Previously, it has been suggested that $40 \mathrm{mg}$ was the recommended afatinib dose for first-line therapy [10]. A recent study has revealed that the PFS of the non-full-dose group was 12.8 months, while the PFS was 


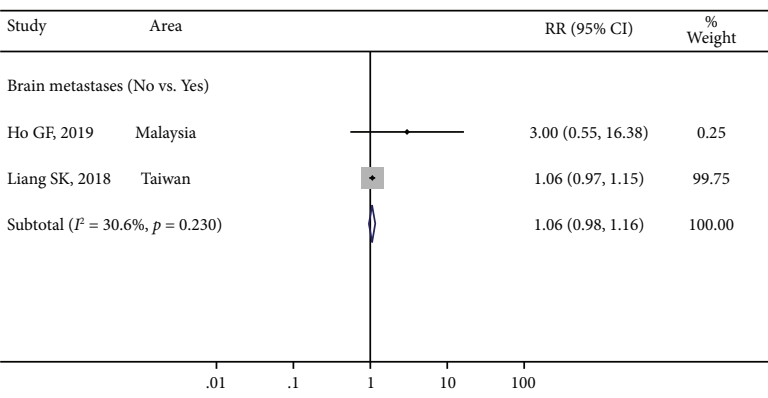

(a)

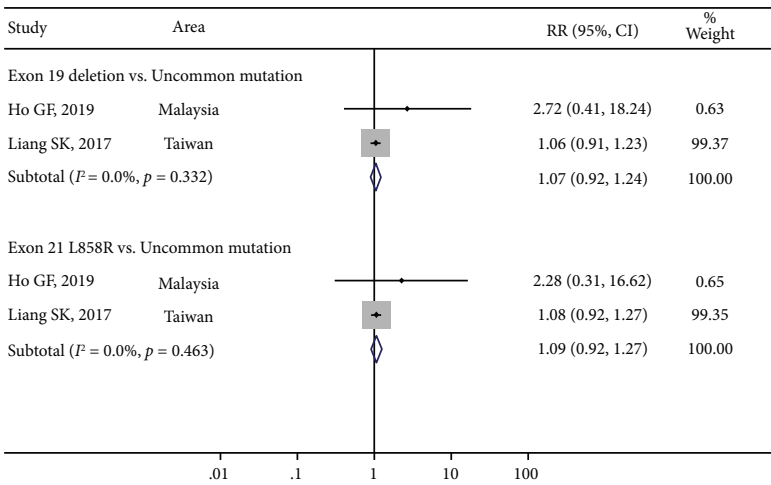

(c)

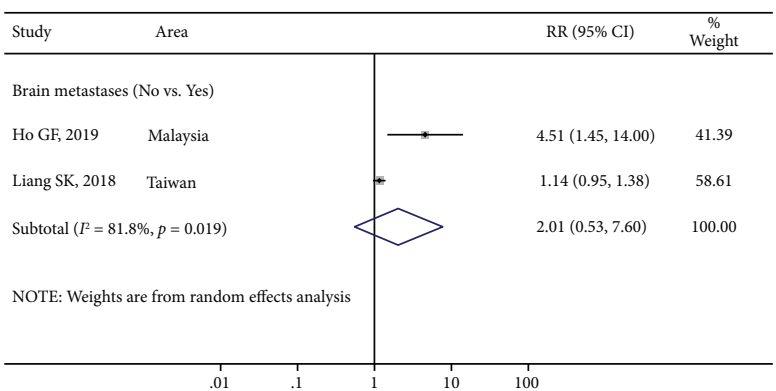

(b)

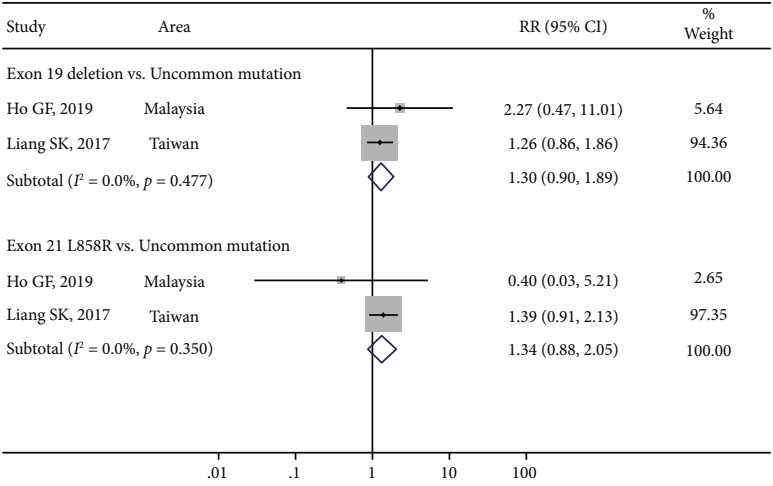

(d)

FIGURE 9: The meta-analysis results for disease control rates (DCRs) and objective response rates (ORRs) in subgroups after afatinib treatment in advanced NSCLC with EGFR mutation: (a) comparison of DCRs in different status of brain metastases; (b) comparison of ORRs in different status of brain metastases; (c) comparison of DCRs in different mutation sites; and (d) comparison of ORRs in different mutation sites.

11.0 months for the full-dose group; however, the difference was not significant (HR: 1.3, 95\% CI [0.9-2.0]) [42]. Yang et al. have reported that afatinib $30 \mathrm{mg}$ daily as an initial dose presents a similar response rate and PFS as an initial dose of $40 \mathrm{mg}$ daily [43]. In the current RWE-based meta-analysis, the results revealed that the difference in PFS and OS between the afatinib non-full-dose group $(<40 \mathrm{mg})$ and fulldose group $(>40 \mathrm{mg})$ was not significant $(P>0.05)$. However, the ORR in the full-dose group was $78.5 \%$ (95\% CI $[66.7 \%, 88.4 \%])$, which was significantly higher than that in the non-full-dose group (67.8\%; 95\% CI [56.8, 77.9]). Thus, the real-world data suggested that decreasing the afatinib dose does not negatively impact efficacy; the full dose should be employed for treating NSCLC patients with EGFR mutations if tolerance permits.

Furthermore, moderate-to-severe adverse drug reactions usually result in dose reduction or discontinuation. $\mathrm{Nu}-$ merous clinical trials have reported that afatinib $40 \mathrm{mg}$ daily as the starting dose presented severe adverse drug reactions, including skin rash, paronychia, and diarrhea [11, 12, 44]. $40 \mathrm{mg}$ afatinib daily presented a significantly higher incidence of Grade 3 skin rash (16\% vs. $0 \%)$ and diarrhea $(100 \%$ vs. $41 \%$ ) than $30 \mathrm{mg}$ daily afatinib [45]. In the present study, the frequency and severity of adverse events (including diarrhea, skin rash, mucositis, paronychia, and pruritus) was higher in patients who administered $40 \mathrm{mg}$ afatinib daily than in those who administered $30 \mathrm{mg}$ afatinib daily. However, the differences of adverse reactions in the two groups of tolerability-guided afatinib dose adjustment were not significant. Moreover, compared with the $40 \mathrm{mg} / \mathrm{day}$ dose, $57.8 \%$ (1917/3319) of patients received a lower afatinib dose, with only $0.5 \%$ (18/3319) of patients receiving a higher afatinib dose; this could partly explain the lower tolerability and higher toxicities associated with afatinib $40 \mathrm{mg}$ daily. However, it should be noted that the anticancer efficacy ORR of afatinib $30 \mathrm{mg}$ daily did not surpass that of the $40 \mathrm{mg}$ daily dose. Besides, the incidence and severity of adverse reactions showed a decreased trend in patients receiving non-full dose, which indicated the tolerability-guided dose adjustment alleviated afatinib-related adverse effects. Thus, the realworld data support that dose adjustment can be guided according to tolerance once adverse reactions occur.

In addition to the afatinib dose, clinical factors such as brain metastases can influence the results of patients with advanced EGFR-mutant NSCLC [46]. In the LUX-Lung 6 trial, the median PFS of patients with brain metastases treated with afatinib was lower than that of patients without brain metastases [47], which was in accordance with our results, suggesting that brain metastases is an influence factor of patients with advanced EGFR-mutant NSCLC based on afatinib dose.

The current study was the first RWE-based meta-analysis to explore the efficacy and adverse reactions in patients with advanced EGFR-mutated NSCLC. However, some limitations persist in the current study: (1) the small sample size of some included studies influenced certain outcome indicators 
of meta-analysis; (2) it was not possible to assess the methodological quality of included studies and the impact of quality on the results in this RWE study owing to a lack of suitable quality evaluation tools; (3) subgroup analysis was not performed on first-generation or second-generation EGFR-TKIs for comparing afatinib with erlotinib, dacomitinib, and gefitinib.

In conclusion, afatinib is a safe and effective first-line treatment in patients with EGFR-mutated NSCLC, and tolerability-guided afatinib dose adjustment might not affect the PFS of these patients. This study was performed based on real-world data, reflecting information on curative effects in real-world patients and fully compensates for disadvantages of RCTs.

$\begin{array}{ll}\text { Abbreviations } \\ \text { CI: } & \text { Confidence interval } \\ \text { DCR: } & \text { Disease control rate } \\ \text { ECOG- } & \text { Eastern Cooperative Oncology Group } \\ \text { PS: } & \text { Performance Status } \\ \text { EGFR: } & \text { Epidermal growth factor receptor } \\ \text { HR: } & \text { Hazard risk } \\ \text { IR: } & \text { Incidence rate } \\ \text { NSCLC: } & \text { Non-small-cell lung cancer } \\ \text { ORR: } & \text { Overall response rate } \\ \text { OS: } & \text { Overall survival } \\ \text { PFS: } & \text { Progression-free survival } \\ \text { RCT: } & \text { Randomized clinical trial } \\ \text { RR: } & \text { Relative risk } \\ \text { RWE: } & \text { Real-world evidence } \\ \text { TTF: } & \text { Time to failure } \\ \text { TKIs: } & \text { Tyrosine kinase inhibitors } \\ \text { WMD: } & \text { Weighted mean difference } \\ \text { AE: } & \text { Adverse event } \\ \text { SAE: } & \text { Severe adverse event. } \\ & \end{array}$

\section{Data Availability}

The raw data supporting the conclusions of this manuscript will be made available by the authors, without undue reservation, to any qualified researcher.

\section{Disclosure}

An earlier version of abstract of this manuscript has been presented as meeting abstract in 2021 ASCO.

\section{Conflicts of Interest}

The authors declare that they have no conflicts of interests.

\section{Authors' Contributions}

Zhang Lemeng, Luo Yongzhong, and Chen Jianhua carried out the conception and design of the research, and Cheng Tianli and Yang Hua participated in the acquisition of data. Pan Changqie, Li Haitao, and Jiang Zhou carried out the analysis and interpretation of data. Zhang Lemeng and Luo Yongzhong participated in the design of the study and prepared and revised the manuscript. All authors read and approved the final manuscript.

\section{Acknowledgments}

This study was supported by grants from the National Natural Science Foundation of Hunan Province (2020JJ4418 and 2020RC3067), Clinical Medical Technology Innovation Guided Project (2020SK51112), Natural Science Foundation of Hunan Province National Health Commission (B2019091 and 20201286), Natural Science Foundation of Changsha Science and Technology Bureau (Kq1901084, Kq1901080, and Kq2001024), Cancer Foundation of China (NCC2018B58), Key Research and Development Project of Hunan Province (2017WK2061), and Hui Lan Public Foundation (HL-HS2020-1).

\section{Supplementary Materials}

Supplementary Figure 1: the meta-analysis results for various incidence rates of adverse events after afatinib treatment in advanced NSCLC with EGFR mutation: (A) the incidence rate of fatigue; (B) the incidence rate of increased alanine aminotransferase (ALT); (C) the incidence rate of increased aspartate aminotransferase (AST) levels; and (D) the incidence rate of interstitial lung disease (ILD). Supplementary Figure 2: the meta-analysis results for incidence rates of severe adverse events after afatinib treatment in advanced NSCLC with EGFR mutation: (A) the adverse reaction incidence rate of fatigue; $(B)$ the adverse reaction incidence rate of increased alanine aminotransferase (ALT) levels; (C) the adverse reaction incidence rate of increased aspartate aminotransferase (AST) levels; and (D) the adverse reaction incidence rate of interstitial lung disease (ILD). Supplementary Figure 3: the meta-analysis results for risk of progression-free survival (PFS) after afatinib treatment in advanced NSCLC with EGFR mutation: (A) PFS between brain metastases group vs. non-brain metastases group and (B) PFS between exon 19 deletion $v s$. uncommon, exon 19 deletion $v s$. exon 21 L858R, brain metastases (no vs. yes), and ECOG-PS (0-1) vs. ECOG-PS $(\geq 2)$. (Supplementary Materials)

\section{References}

[1] No authors listed, "Erratum: Global cancer statistics 2018: GLOBOCAN estimates of incidence and mortality worldwide for 36 cancers in 185 countries," CA: A Cancer Journal for Clinicians, vol. 70, p. 313, 2020.

[2] R. S. Herbst, D. Morgensztern, and C. Boshoff, "The biology and management of non-small cell lung cancer," Nature, vol. 553, no. 7689, pp. 446-454, 2018.

[3] Y. Shi, J. S.-K. Au, S. Thongprasert et al., "A prospective, molecular epidemiology study of EGFR mutations in Asian patients with advanced non-small-cell lung cancer of adenocarcinoma histology (PIONEER)," Journal of Thoracic Oncology, vol. 9, no. 2, pp. 154-162, 2014.

[4] P. T. Harrison, S. Vyse, and P. H. Huang, "Rare epidermal growth factor receptor (EGFR) mutations in non-small cell lung cancer," Seminars in Cancer Biology, vol. 61, pp. 167-179, 2020. 
[5] B.-C. Liao, C.-C. Lin, and J. C.-H. Yang, "Novel EGFR inhibitors in non-small cell lung cancer: current status of afatinib," Current Oncology Reports, vol. 19, no. 1, p. 4, 2017.

[6] R. T. Dungo and G. M. Keating, "Afatinib: first global approval," Drugs, vol. 73, no. 13, pp. 1503-1515, 2013.

[7] E. S. Kim, B. Halmos, I. F. Kohut et al., "Efficacy and safety results of the afatinib expanded access program," Oncology and Therapy, vol. 5, no. 1, pp. 103-110, 2017.

[8] J. C.-H. Yang, V. Hirsh, M. Schuler et al., "Symptom control and quality of life in LUX-Lung 3: a phase III study of afatinib or cisplatin/pemetrexed in patients with advanced lung adenocarcinoma with EGFR mutations," Journal of Clinical Oncology, vol. 31, no. 27, pp. 3342-3350, 2013.

[9] M. Schuler, L. Paz-Ares, L. V. Sequist et al., "First-line afatinib for advanced EGFRm+ NSCLC: analysis of long-term responders in the LUX-Lung 3, 6, and 7 trials," Lung Cancer, vol. 133, pp. 10-19, 2019.

[10] C.-Y. Liu, C.-L. Wang, S.-H. Li et al., "The efficacy of $40 \mathrm{mg}$ versus dose de-escalation to less than $40 \mathrm{mg}$ of afatinib (Giotrif) as the first-line therapy for patients with primary lung adenocarcinoma harboring favorable epidermal growth factor mutations," Oncotarget, vol. 8, no. 57, pp. 97602-97612, 2017.

[11] L. V. Sequist, J. C.-H. Yang, N. Yamamoto et al., "Phase III study of afatinib or cisplatin plus pemetrexed in patients with metastatic lung adenocarcinoma with EGFR mutations," Journal of Clinical Oncology, vol. 31, no. 27, pp. 3327-3334, 2013.

[12] K. Park, E.-H. Tan, K. O’Byrne et al., "Afatinib versus gefitinib as first-line treatment of patients with EGFR mutation-positive non-small-cell lung cancer (LUX-Lung 7): a phase 2B, open-label, randomised controlled trial," The Lancet Oncology, vol. 17, no. 5, pp. 577-589, 2016.

[13] Y. Zhao, J. Liu, X. Cai et al., "Efficacy and safety of first line treatments for patients with advanced epidermal growth factor receptor mutated, non-small cell lung cancer: systematic review and network meta-analysis," BMJ, vol. 367, p. 15460, 2019.

[14] J. Han, J.-z. Wang, X. Yang et al., "METTL3 promote tumor proliferation of bladder cancer by accelerating pri-miR221/ 222 maturation in m6A-dependent manner," Molecular Cancer, vol. 18, no. 1, p. 110, 2019.

[15] Z. Yang, A. Hackshaw, Q. Feng et al., "Comparison of gefitinib, erlotinib and afatinib in non-small cell lung cancer: a meta-analysis," International Journal of Cancer, vol. 140, no. 12, pp. 2805-2819, 2017.

[16] J. C.-H. Yang, L. V. Sequist, C. Zhou et al., "Effect of dose adjustment on the safety and efficacy of afatinib for EGFR mutation-positive lung adenocarcinoma: post hoc analyses of the randomized LUX-Lung 3 and 6 trials," Annals of Oncology, vol. 27, no. 11, pp. 2103-2110, 2016.

[17] G. Nabi, "Randomised controlled trials in medical research: do we need alternatives?" Scottish Medical Journal, vol. 65, no. 1, pp. 1-2, 2020.

[18] S. Monti, V. Grosso, M. Todoerti, and R. Caporali, "Randomized controlled trials and real-world data: differences and similarities to untangle literature data," Rheumatology, vol. 57, no. Supplement_7, pp. vii54-vii58, 2018.

[19] K. Park, D. Wan-Teck Lim, I. Okamoto, and J. C. Yang, "Firstline afatinib for the treatment of EGFR mutation-positive non-small-cell lung cancer in the "real-world" clinical setting," Therapeutic advances in medical oncology, vol. 11, Article ID 1758835919836374, 2019.

[20] B. Halmos, E.-H. Tan, R. A. Soo et al., "Impact of afatinib dose modification on safety and effectiveness in patients with
EGFR mutation-positive advanced NSCLC: results from a global real-world study (RealGiDo)," Lung Cancer, vol. 127, pp. 103-111, 2019.

[21] L. Shamseer, D. Moher, M. Clarke et al., "Preferred reporting items for systematic review and meta-analysis protocols (PRISMA-P) 2015: elaboration and explanation," BMJ, vol. 349, no. 1, p. g7647, 2015.

[22] S. Popat, A. Matakidou, and R. S. Houlston, "Thymidylate synthase expression and prognosis in colorectal cancer: a systematic review and meta-analysis," Journal of Clinical Oncology, vol. 22, no. 3, pp. 529-536, 2004.

[23] J. P. T. Higgins, S. G. Thompson, J. J. Deeks, and D. G. Altman, "Measuring inconsistency in meta-analyses," BMJ, vol. 327, no. 7414 , pp. 557-560, 2003.

[24] J. A. Sterne and M. Egger, "Regression methods to detect publication and other bias in meta-analysis," Publication Bias in Meta-Analysis: Prevention, Assessment and Adjustments, pp. 99-110, 2005.

[25] K. Brat, M. Bratova, J. Skrickova et al., "Real-life effectiveness of first-line anticancer treatments in stage IIIB/IV NSCLC patients: data from the C zech TULUNG R egistry," Thoracic Cancer, vol. 11, no. 11, pp. 3346-3356, 2020.

[26] W. Su, H. H. Han, Y. Wang et al., "The polycomb repressor complex 1 drives double-negative prostate cancer metastasis by coordinating stemness and immune suppression," Cancer Cell, vol. 36, no. 2, pp. 139-155, 2019.

[27] M. Del Re, I. Petrini, F. Mazzoni et al., "Incidence of T790M in patients with NSCLC progressed to gefitinib, erlotinib, and afatinib: a study on circulating cell-free dna," Clinical Lung Cancer, vol. 21, no. 3, pp. 232-237, 2020.

[28] G.-F. Ho, C.-S. Chai, A. Alip et al., "Real-world experience of first-line afatinib in patients with EGFR-mutant advanced NSCLC: a multicenter observational study," BMC Cancer, vol. 19, no. 1, p. 896, 2019.

[29] Y. Y. Hsieh, W. T. Fang, Y. W. Lo, Y. H. Chen, and L. N. Chien, "Comparing the effectiveness of different EGFRTKIs in patients with EGFR mutant non-small-cell lung cancer: a retrospective cohort study in Taiwan," International Journal of Cancer, vol. 147, no. 4, pp. 1107-1116, 2020.

[30] S. Igawa, T. Ono, M. Kasajima, S. Kusuhara, S. Otani, and T. Fukui, "Real-world assessment of afatinib for patients with EGFR-positive non-small cell lung cancer," Investigational New Drugs, vol. 38, no. 6, pp. 1906-1914, 2020.

[31] K. Ito, K. Murotani, A. Kubo et al., "Propensity score analysis of overall survival between first- and second-generation EGFR-TKIs using real-world data," Cancer Science, vol. 111, no. 10, pp. 3705-3713, 2020.

[32] F. Khan, C. Ottensmeier, S. Popat et al., "Afatinib use in nonsmall cell lung cancer previously sensitive to epidermal growth factor receptor inhibitors: the United Kingdom Named Patient Programme," European Journal of Cancer, vol. 50, no. 10, pp. 1717-1721, 2014.

[33] !!! INVALID CITATION !!!!

[34] A. Fujiwara, M. Yoshida, H. Fujimoto et al., "A retrospective comparison of the clinical efficacy of gefitinib, erlotinib, and afatinib in Japanese patients with non-small cell lung cancer," Oncology Research Featuring Preclinical and Clinical Cancer Therapeutics, vol. 26, no. 7, pp. 1031-1036, 2018.

[35] M. J. Hochmair, A. Morabito, D. Hao et al., "Sequential treatment with afatinib and osimertinib in patients with EGFR mutation-positive non-small-cell lung cancer: an observational study," Future Oncology, vol. 14, no. 27, pp. 2861-2874, 2018.

[36] H. A. Jung, S. Park, J. M. Sun, S. H. Lee, J. S. Ahn, and M. J. Ahn, "Treatment and outcomes of metastatic non-small- 
cell lung cancer harboring uncommon EGFR mutations: are they different from those with common EGFR mutations?" Biology, vol. 9, 2020.

[37] M. Kanazu, M. Mori, M. Kimura, K. Nishino, T. Shiroyama, and I. Nagatomo, "Effectiveness of EGFR tyrosine kinase inhibitors in advanced non-small cell lung cancer patients with uncommon EGFR mutations: a multicenter observational study," Thorac Cancer, vol. 12, no. 1, 2020.

[38] F.-C. Kuan, S.-H. Li, C.-L. Wang, M.-H. Lin, Y.-H. Tsai, and C.-T. Yang, "Analysis of progression-free survival of first-line tyrosine kinase inhibitors in patients with non-small cell lung cancer harboring leu858Arg or exon 19 deletions," Oncotarget, vol. 8, no. 1, pp. 1343-1353, 2017.

[39] J. Lim, C. Samuelsen, A. Golembesky, S. Shrestha, L. Wang, and I. Griebsch, "Duration of treatment among patients prescribed afatinib or erlotinib as first-line therapy for EGFR mutation-positive non-small-cell lung cancer in the USA," Future Oncology, vol. 15, no. 13, pp. 1493-1504, 2019.

[40] V. Y.-F. Su, K.-Y. Yang, T.-Y. Huang et al., "The efficacy of first-line tyrosine kinase inhibitors combined with co-medications in Asian patients with EGFR mutation non-small cell lung cancer," Scientific Reports, vol. 10, no. 1, Article ID 14965, 2020.

[41] S.-G. Wu, C.-L. Chiang, C.-Y. Liu et al., "An observational study of acquired EGFR t790m-dependent resistance to EGFR-TKI treatment in lung adenocarcinoma patients in taiwan," Frontiers in Oncology, vol. 10, p. 1481, 2020.

[42] Y. Iida, F. Kumasawa, T. Shimizu, Y. Shintani, N. Takahashi, and Y. Gon, "Successful treatment of an elderly patient with an uncommon L861Q epidermal growth factor receptor mutation with low-dose afatinib: a case report," Thoracic Cancer, vol. 11, no. 2, pp. 447-450, 2020.

[43] C.-J. Yang, M.-J. Tsai, J.-Y. Hung et al., "The clinical efficacy of Afatinib $30 \mathrm{mg}$ daily as starting dose may not be inferior to Afatinib $40 \mathrm{mg}$ daily in patients with stage IV lung Adenocarcinoma harboring exon 19 or exon 21 mutations," BMC Pharmacology and Toxicology, vol. 18, no. 1, p. 82, 2017.

[44] Y.-L. Wu, C. Zhou, C.-P. Hu et al., "Afatinib versus cisplatin plus gemcitabine for first-line treatment of Asian patients with advanced non-small-cell lung cancer harbouring EGFR mutations (LUX-Lung 6): an open-label, randomised phase 3 trial," The Lancet Oncology, vol. 15, no. 2, pp. 213-222, 2014.

[45] L. Paz-Ares, E.-H. Tan, K. O’Byrne et al., "Afatinib versus gefitinib in patients with EGFR mutation-positive advanced non-small-cell lung cancer: overall survival data from the phase IIb LUX-Lung 7 trial," Annals of Oncology, vol. 28, no. 2, pp. 270-277, 2017.

[46] W.-L. Tan, Q. S. Ng, C. Lim et al., "Influence of afatinib dose on outcomes of advanced EGFR-mutant NSCLC patients with brain metastases," BMC Cancer, vol. 18, no. 1, p. 1198, 2018.

[47] M. Schuler, Y.-L. Wu, V. Hirsh et al., "First-line afatinib versus chemotherapy in patients with non-small cell lung cancer and common epidermal growth factor receptor gene mutations and brain metastases," Journal of Thoracic Oncology, vol. 11, no. 3, pp. 380-390, 2016. 